\title{
The Two-Phase Hele-Shaw Problem with a Nonregular Initial Interface and Without Surface Tension
}

\author{
B.V. Bazaliy and N. Vasylyeva \\ Institute of Applied Mathematics and Mechanics \\ 74 R. Luxemburg Str., Donetsk 83114, Ukraine \\ E-mail: bazaliy@iamm.ac.donetsk.ua \\ vasylyeva@iamm.ac.donetsk.ua
}

Received November 6, 2012, revised May 28, 2013

\begin{abstract}
In the paper, we consider the two-dimensional Muskat problem without surface tension on a free boundary. The initial shape of the unknown interface has a corner point. We prove that the problem has a unique solution in the weighted Hölder classes locally in time and specify the sufficient conditions for the existence of the "waiting time" phenomenon.

Key words: Laplace equation, free boundary problems, Muskat problem, weighted Hölder spaces, waiting time property.

Mathematics Subject Classification 2010: 35R35 (primary); 35J25, 35B65 (secondary).
\end{abstract}

\section{Introduction}

The two-phase Hele-Shaw problem (the Muskat problem) describes the evolution of an interface between two immiscible incompressible fluids (for example, water and oil). The motion of fluids is governed by the Darcy law, stating that the velocities of fluids are proportional to the pressure gradients, and the law of mass conservation [25]. The Muskat problem with a regular initial interface was studied by L. Jiang and Y. Chen [19], F. Yi [30, 31], F. Otto [26], S. Howison [18], D. Ambrose [1], M. Siegel, R. Caflish and S. Howison [28], S.P. Degtyarev [12], J. Escher and B.V. Matioc [14].

Weak and variational solutions for the one-phase Hele-Shaw problem were studied by C. Elliott and J.R. Ockendon [13], E. Di Benedetto and A. Friedman [6]. Y.E. Hohlov and S.Howison [17] constructed explicit solutions to the HeleShaw problem. B.V. Bazaliy [4] and J. Escher and G. Simonett [15] proved the existence of classical solutions to the Hele-Shaw problem with regular initial data. Preliminary arguments show that if the initial interface in the one-phase 
Hele-Shaw problem has an angle point, then the behaviour of the free boundary depends on the angle value. Moreover, J.R. King, A.A. Lacey, and J.L. Vazquez [20] found that under certain sufficient conditions the angle value is preserved for some time (the "waiting time" phenomenon). In [29], N. Vasylyeva gave a strong proof of the solvability in the weighted Hölder classes for the one-phase Hele-Shaw problem with the "waiting time" property. The similar problem with surface tension was considered by A. Friedman and B.V. Bazaliy [5]. We remark that introduction of the surface tension in one- or two-phase Hele-Shaw problems leads to the regularization of the free boundary problem. The surface tension variation of the Muskat problem was previously studied by us in [7]. In the present paper we apply the same method, but the model problem corresponding to the angle point of the initial interface and the related linear equation turned out to be more complicated. As in [7], our purpose is to formulate a set of sufficient conditions under which the problem has a solution in the weighted Hölder classes with the "waiting time" property.

The paper is organized as follows. In Section 2, we formulate our problem, reduce the problem with unknown boundary to a problem in a fixed domain, define the weighted Hölder spaces and state the main result, Theorem 2.1. In Subsection 2.4, we represent our problem in the form $\Im \mathbf{z}=f(x, t)+\mathrm{F}_{1}(\mathbf{z})$, where $\mathbf{z}=\left(\theta_{1}, \theta_{2}, \sigma\right)$ and $\Im$ is a linear operator, the vector $f(x, t)$ is constructed by using initial data, and $F_{1}$ is a nonlinear operator. Section 3 is devoted to studying the model problems in the plane corners and in the half-spaces. Then, in Section 4, using the technique of the regularizer for parabolic systems [22] together with the results of Section 3, we prove the one-to-one solvability to the linear problem $\Im \mathbf{z}=f(x, t)$, Theorem 4.1. In Section 5 , we prove the main results by using Theorem 4.1 and the fixed point theorem.

\section{The Statement of the Problem and the Main Result}

\subsection{The mathematical model}

Let $\Omega$ be a double-connected bounded open domain in $R^{2}$ with the boundary $\partial \Omega=\Gamma_{1} \bigcup \Gamma_{2}, \Gamma_{1} \bigcap \Gamma_{2}=\emptyset$ (see Figure 1 below). Let $\Gamma(t)$, for each $t \in[0, T]$, be a simple closed curve $\Gamma(t) \subset \Omega$ that separates $\Omega$ into two subdomains $\Omega_{1}(t)$ and $\Omega_{2}(t)$ such that $\Omega=\Omega_{1}(t) \bigcup \Gamma(t) \bigcup \Omega_{2}(t)$, and $\partial \Omega_{i}=\Gamma_{i} \bigcup \Gamma(t), i=1,2$.

In the two-phase Hele-Shaw problem we are looking for the fluid domain $\Omega_{i}(t)$ and the fluid pressure $p_{i}(y, t), y \in \Omega_{i}(t), t \in[0, T], i=1,2$, such that

$$
\begin{gathered}
\Delta_{y} p_{i}=0 \quad \text { in } \quad \Omega_{i}(t), i=1,2, t \in[0, T], \\
p_{1}-p_{2}=0 \quad \text { on } \Gamma(t), \\
V_{n}=-k_{1} \frac{\partial p_{1}}{\partial n}=-k_{2} \frac{\partial p_{2}}{\partial n} \quad \text { on } \quad \Gamma(t),
\end{gathered}
$$




$$
\begin{gathered}
p_{i}=\psi_{i}(y) \quad \text { on } \quad \Gamma_{i T}=\Gamma_{i} \times[0, T], \\
\Omega_{i}(0), \quad \Gamma(0) \quad \text { are given } .
\end{gathered}
$$

Here $\Delta_{y}=\frac{\partial^{2}}{\partial y_{1}^{2}}+\frac{\partial^{2}}{\partial y_{2}^{2}}, n$ is the normal to $\Gamma(t)$ directed in $\Omega_{1}(t), V_{n}$ is the velocity of points $\Gamma(t)$ in the direction of $n ; k_{1}$ and $k_{2}$ are positive constants, $k_{i}=\frac{\bar{k}}{\mu_{i}}$, where $\bar{k}=$ const $>0$ is the permeability of the porous medium and $\mu_{i}$ is the fluid viscosity in $\Omega_{i}(t), i=1,2, \mu_{i}$ are positive constants; $\psi_{i}(y)$ are given positive functions.

If we consider, for example, a physical problem where $\Omega_{1}(t)$ is occupied by water and $\Omega_{2}(t)$ is filled by oil, then $\mu_{2}>\mu_{1}$, and hence, $k=\frac{k_{2}}{k_{1}}<1$.

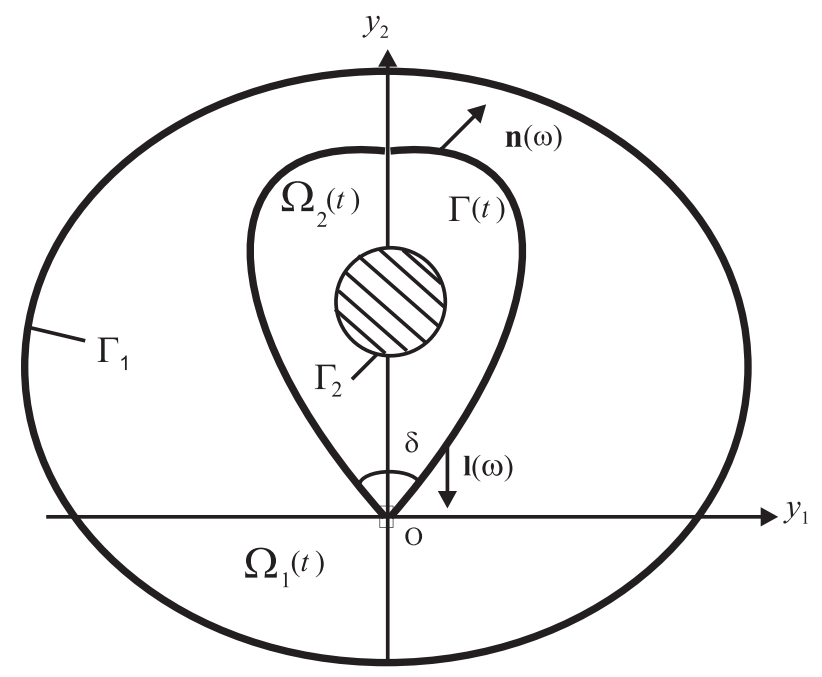

Fig. 1. Problem (M)

We will suppose that $\Gamma(0)$ has an angle point of opening $\delta, \delta \in(0, \pi)$, and the origin of the coordinate system $\left(y_{1}, y_{2}\right)$ is placed at the vertex of this corner (see Figure 1). For the sake of simplicity, we consider problem (2.1)-(2.5) under the assumption that $\Omega_{i}(0)$ and $\Gamma(0)$ are symmetric with respect to the $y_{2}-$ axis, $\psi_{i}(y)$ are even functions in $y_{1}$, and we seek a symmetric solution with the condition

$$
\left.\frac{\partial p_{i}}{\partial y_{1}}\right|_{y_{1}=0}=0, \quad i=1,2 .
$$

One can see that (2.1) and (2.2), together with the second equality in (2.3), define the transition problem with the interface $\Gamma(t)$, and the first equality in (2.3) serves to find the unknown curve $\Gamma(t)$ that is called the free boundary.

We denote the Muscat problem (2.1)-(2.6) as Problem (M). 


\subsection{Reducing to a fixed domain}

As in [5] and [7], we use the Hanzawa method [16] to reduce the free boundary Muskat problem to a problem in a fixed domain. Let $\Omega_{i}(0) \cap\left\{y_{1}>0\right\}=\Omega_{i}$, $\Gamma=\Gamma(0) \cap\left\{y_{1} \geq 0\right\}, \Gamma \in C^{\varrho+\alpha}, \varrho$ be an integer and $\varrho \geq 3, \alpha \in(0,1)$, and $\omega$ be some parameter along $\Gamma$ (for example, the arc length of $\Gamma$ ). The position of a point on $\Gamma$ we define as $\bar{m}(\omega)$. Let $n(\omega)$ be the normal to $\Gamma$ directed in $\Omega_{1}$, and $\bar{l}(\omega)$ be the $C^{\varrho+\alpha}(\varrho \geq 2)$ vector field on $\Gamma$ which is transversal to $\Gamma$ such that $\bar{l}(\omega)=(0,-1)$ in the $\varepsilon_{0}$-neighborhood of $O=(0,0)$ and $\bar{l}(\omega)=n(\omega)$ out of the $2 \varepsilon_{0}$-neighborhood of $O$.

For sufficiently small $\gamma_{0}>0$, $\omega$-lines: $\bar{m}(\omega)+\eta \bar{l}(\omega),|\eta|<2 \gamma_{0}$, do not intersect each other, and $\Gamma_{1} \cup \Gamma_{2}$. The mapping $(\omega, \eta) \rightarrow y=y(\omega, \eta)$ defined by

$$
y=\left(y_{1}, y_{2}\right)=\bar{m}(\omega)+\eta \bar{l}(\omega)
$$

is a diffeomorphism from $M=W \times\left(-\gamma_{0}, \gamma_{0}\right)$ onto

$$
N=\{y: y=\bar{m}(\omega)+\eta \bar{l}(\omega),(\omega, \eta) \in M\} .
$$

The inverse mapping $\Sigma: N \rightarrow M$ is

$$
\Sigma: y \rightarrow(\omega(y), \eta(y)) .
$$

We assume that the free boundary in Problem (M) has the form

$$
\Gamma_{\rho}(t)=\{(y, t): y(\omega, t)=\bar{m}(\omega)+\rho(\omega, t) \bar{l}(\omega), t \in[0, T]\},
$$

where $|\rho(\omega, t)|<\gamma_{0} / 4, \rho(\omega, 0)=0$. It means that the free boundary equation is given by

$$
\Phi_{\rho}(y, t)=\eta(y)-\rho(\omega, t)=0,(y, t) \in N \times[0, T] .
$$

The surface $\Gamma_{\rho}(t)$ splits $\Omega_{T}=\Omega \times[0, T]$ into domains $\Omega_{i}(t)$. Let $\chi(\lambda) \in C_{0}^{\infty}\left(R^{1}\right)$, $\chi(\lambda)=1$ if $|\lambda|<\gamma_{0} / 3$ and $\chi(\lambda)=0$ if $|\lambda|>\gamma_{0},\left|\chi^{\prime}\right| \leq$ const $/ \gamma_{0}$, const $<2$. We will use the coordinates $(\omega, \eta)$ to define the diffeomorphism

$$
e_{\rho}:(x, t) \rightarrow(y, t)
$$

from $X_{T}=R^{2} \times[0, T]$ onto $Y_{T}=R^{2} \times[0, T]$ by setting

$$
\left\{\begin{array}{l}
\omega(y)=\omega(x), \\
\eta(y)=\lambda(x)+\chi(\lambda(x)) \rho(\omega(x), t), \text { if }(\omega(x), \lambda(x)) \in N, \\
y=x, \text { otherwise, }
\end{array}\right.
$$

such that the transform $e_{\rho}^{-1}$ maps $\Omega_{i}(t)$ onto $\Omega_{i} \times[0, T]=\Omega_{i T}$ and $\Gamma_{\rho}(t)$ onto $\Gamma \times[0, T]=\Gamma_{T}$, the free boundary is given by $e_{\rho}(\{\lambda(x)=0\})$, and $\omega(x), \lambda(x)$ are 
the coordinates in $X_{T}$ similar to the coordinates $\omega(y), \eta(y)$ in $Y_{T}$. The change of variables gives the new desired functions

$$
v_{i}\left(x_{1}, x_{2}, t\right)=p_{i}(y, t) \circ e_{\rho}(x, t), i=1,2,
$$

which satisfy the equations

$$
\begin{gathered}
\nabla_{\rho}^{2} v_{i}(x, t)=0 \quad \text { in } \quad \Omega_{i T}, \\
v_{1}(x, t)-v_{2}(x, t)=0 \quad \text { on } \quad \Gamma_{T}, \\
v_{i}=\psi_{i}(x) \quad \text { on } \quad \Gamma_{i T}, \\
\frac{\partial v_{i}}{\partial x_{1}}=0 \quad \text { on } \quad x_{1}=0,
\end{gathered}
$$

where we take into account that $y=x$ near $\Gamma_{i T}$. Here $\nabla_{\rho}=\left(E_{\rho}^{*}\right)^{-1} \nabla_{x}$, where $E_{\rho}$ is the Jacobi matrix of the mapping $y=e_{\rho}(x, t), \nabla_{x}=\left(\frac{\partial}{\partial x_{1}}, \frac{\partial}{\partial x_{2}}\right)$. It follows from (2.7) that the unit normal to $\Gamma_{\rho}(t)$ is

and therefore

$$
n=\frac{\nabla_{y} \Phi_{\rho}}{\left|\nabla_{y} \Phi_{\rho}\right|},
$$

$$
V_{n}=-\frac{\frac{\partial \Phi_{\rho}}{\partial t}}{\left|\nabla_{y} \Phi_{\rho}\right|}=\frac{\frac{\partial \rho(\omega, t)}{\partial t}}{\left|\nabla_{y} \Phi_{\rho}\right|} .
$$

Now we can conclude that equation (2.3) takes the form

$$
\begin{gathered}
\frac{\partial \rho(\omega, t)}{\partial t}=-k_{1}\left(\nabla_{y} p_{1}, \nabla_{y} \Phi_{\rho}\right)=-k_{2}\left(\nabla_{y} p_{2}, \nabla_{y} \Phi_{\rho}\right), \\
\rho(\omega, 0)=0 .
\end{gathered}
$$

Since $\Phi_{\rho}=0$ on $\Gamma(t)$, we get

$$
\left(\nabla_{y} p_{i}, \nabla_{y} \Phi_{\rho}\right)=\left(\nabla_{\rho} v_{i}, \nabla_{\rho} \Phi_{\rho}\right)=S\left(\omega, \rho, \rho_{\omega}\right) \frac{\partial v_{i}}{\partial \lambda}+S_{1}\left(\omega, \rho, \rho_{\omega}\right) \frac{\partial v_{i}}{\partial \omega},
$$

where $S\left(\omega, \rho, \rho_{\omega}\right), S_{1}\left(\omega, \rho, \rho_{\omega}\right)$ are some specific smooth functions

$$
S\left(\omega, \rho, \rho_{\omega}\right)=\left(\nabla_{\rho} \lambda, \nabla_{\rho} \lambda\right), S_{1}\left(\omega, \rho, \rho_{\omega}\right)=\left(\nabla_{\rho} \omega, \nabla_{\rho} \lambda\right) .
$$

Thus our initial free boundary Muskat problem is reduced to the problem in the fixed domain for the functions $v_{i}\left(x_{1}, x_{2}, t\right), i=1,2$, and $\rho(\omega, t)$ that satisfy equations (2.10)-(2.15). We denote this problem as $\left(M_{1}\right)$ :

$$
\begin{gathered}
\nabla_{\rho}^{2} v_{i}(x, t)=0 \quad \text { in } \quad \Omega_{i T}, i=1,2, \\
v_{1}(x, t)-v_{2}(x, t)=0 \quad \text { on } \quad \Gamma_{T}, \\
-\rho_{t}(\omega, t)=k_{1}\left(\nabla_{\rho} v_{1}, \nabla_{\rho} \Phi_{\rho}\right)=k_{2}\left(\nabla_{\rho} v_{2}, \nabla_{\rho} \Phi_{\rho}\right) \quad \text { on } \Gamma_{T}, \\
v_{i}=\psi_{i}(x) \quad \text { on } \quad \Gamma_{i T},\left.\quad \frac{\partial v_{i}}{\partial x_{1}}\right|_{x_{1}=0}=0, \quad \rho(\omega, 0)=0 .
\end{gathered}
$$




\subsection{Weighted Hölder spaces and the main result}

Let $D$ be a given domain in $R^{2}$ with a corner point at the origin of coordinates and let $D_{T}=D \times[0, T]$. Denote the distance from the origin of coordinates to the point $y \in \bar{D}$ by $r(y)$. We set $r(y, x)=\min \{r(y), r(x)\}, x, y \in \bar{D}$. Let $s$ be a given number, $\varrho$ be an integer, $\varrho \geq 0, \alpha \in(0,1)$. The Banach space $E_{s}^{\varrho+\alpha, \alpha, \alpha}\left(\bar{D}_{T}\right)$ is the set of the functions $u(x, t)$ with the finite norm

$$
\begin{gathered}
\|u\|_{E_{s}^{\varrho+\alpha, \alpha, \alpha}\left(\bar{D}_{T}\right)}=\sum_{|l|=0}^{\varrho}\left[\sup _{\bar{D}_{T}} r^{|l|-s}(x)\left|D_{x}^{l} u(x, t)\right|+\left\langle D_{x}^{l} u(x, t)\right\rangle_{x, s-|l|, D_{T}}^{(\alpha)}\right. \\
\left.+\left\langle D_{x}^{l} u(x, t)\right\rangle_{t, s-|l|, D_{T}}^{(\alpha)}+\left[D_{x}^{l} u(x, t)\right]_{s-|l|, D_{T}}^{(\alpha, \alpha)}\right]
\end{gathered}
$$

where

$$
\begin{gathered}
\langle u\rangle_{x, s, D_{T}}^{(\alpha)}=\begin{array}{l}
\sup _{(\bar{x}, t),(x, t) \in \bar{D}_{T},} r^{\alpha-s}(x, \bar{x}) \frac{|u(\bar{x}, t)-u(x, t)|}{|x-\bar{x}|^{\alpha}}, \\
|x-\bar{x}|<r(x, \bar{x}) / 2
\end{array} \\
\langle u\rangle_{t, s, D_{T}}^{(\alpha)}=\sup _{(x, t),(x, \tau) \in \bar{D}_{T}} r^{-s}(x) \frac{|u(x, t)-u(x, \tau)|}{|t-\tau|^{\alpha}},
\end{gathered}
$$

and

$$
\begin{aligned}
& {[u]_{s, D_{T}}^{(\alpha, \alpha)}=\sup _{\bar{x}, x \in \bar{D}, t, \tau \in[0, T],} r^{\alpha-s}(x, \bar{x}) \frac{|u(\bar{x}, t)-u(x, t)-u(\bar{x}, \tau)+u(x, \tau)|}{|\bar{x}-x|^{\alpha}|t-\tau|^{\alpha}} .} \\
& |x-\bar{x}|<r(x, \bar{x}) / 2
\end{aligned}
$$

We introduce the space $E_{s}^{\varrho+\alpha, \alpha, \alpha}\left(\overline{\partial D}_{T}\right)$ in a similar way. For the functions $u(x)$ independent of $t$ we use the space $E_{s}^{\varrho+\alpha}(\bar{D})$ with the finite norm

$$
\|u\|_{E_{s}^{\varrho+\alpha}(\bar{D})}=\sum_{|l|=0}^{\varrho}\left[\sup _{\bar{D}} r^{|l|-s}(x)\left|D_{x}^{l} u(x)\right|+\left\langle D_{x}^{l} u(x)\right\rangle_{x, s-|l|, D}^{(\alpha)}\right],
$$

where

$$
\langle u\rangle_{x, s, D}^{(\alpha)}=\sup _{\substack{\bar{x}, x \in \bar{D},|x-\bar{x}|<r(x, \bar{x}) / 2}} r^{\alpha-s}(x, \bar{x}) \frac{|u(\bar{x})-u(x)|}{|x-\bar{x}|^{\alpha}} .
$$

If the domain $D$ does not contain a corner point, the definition of the space $E_{s}^{\varrho+\alpha, \alpha, \alpha}\left(\bar{D}_{T}\right)$ remains as before with $r(x) \equiv 1$. In this case we use the notation $E^{\varrho+\alpha, \alpha, \alpha}\left(\bar{D}_{T}\right)$. 
For the functions $f(\omega, t)$ defined on $\Gamma_{T}$, we will use the weighted Hölder space $N_{s, \gamma}^{2+\alpha}\left(\Gamma_{T}\right)$ with the norm

$$
\|f\|_{N_{s, \gamma}^{2+\alpha}\left(\Gamma_{T}\right)}=\left\|r^{1+\gamma} f\right\|_{E_{s}^{2+\alpha, \alpha, \alpha}\left(\bar{\Gamma}_{T}\right)}+\left\|f_{t}\right\|_{E_{s-1}^{1+\alpha, \alpha, \alpha}\left(\bar{\Gamma}_{T}\right)}
$$

where $\gamma$ is some positive number.

We define the functions $v_{i 0}(x)=v_{i}(x, 0)$ as a solution of the transmission problem

$$
\begin{gathered}
\Delta v_{i 0}=0 \quad \text { in } \quad \Omega_{i}, i=1,2, \\
v_{10}-v_{20}=0, k_{1} \frac{\partial v_{10}}{\partial n}=k_{2} \frac{\partial v_{20}}{\partial n} \text { on } \Gamma, \\
v_{i 0}=\psi_{i}(x) \text { on } \quad \Gamma_{i},\left.\frac{\partial v_{i 0}}{\partial x_{1}}\right|_{x_{1}=0}=0 .
\end{gathered}
$$

By Theorem 1.1 and Remark 3.1 from [8], there exists a unique solution $\left(v_{10}(x), v_{20}(x)\right)$ to problem $(2.17)$, and

$$
\left\|v_{i 0}\right\|_{E_{2+\gamma}^{3+\alpha}\left(\bar{\Omega}_{i}\right)} \leq \operatorname{const}\left(\left\|\psi_{1}\right\|_{E^{3+\alpha}\left(\bar{\Gamma}_{1}\right)}+\left\|\psi_{2}\right\|_{E^{3+\alpha}\left(\bar{\Gamma}_{2}\right)}\right),
$$

where $\alpha \in(0,1)$, and

$$
\begin{gathered}
\gamma \in\left(1+\frac{1}{2}, 1+\frac{\pi+3 \delta}{2 \pi-\delta}\right) \quad \text { if } \quad \delta \in(0, \pi / 7) ; \\
\gamma \in\left(1+\frac{3 \delta}{\pi-\delta}, 1+\frac{\pi+3 \delta}{2 \pi-\delta}\right) \quad \text { if } \quad \delta \in(\pi / 7, \pi / 4) .
\end{gathered}
$$

Theorem 2.1. Let $k=\frac{k_{2}}{k_{1}}, \alpha \in(0,1 / 2), \psi_{i} \in C^{3+\alpha}\left(\bar{\Gamma}_{i}\right), i=1,2 ; \Gamma$ and $\Gamma_{i} \in C^{3+\alpha}$ satisfy the assumptions mentioned in Subsections 2.1 and 2.2; the initial pressures $\left(v_{10}(x), v_{20}(x)\right)$ be given with (2.17) and inequality (2.18) hold;

$$
0<k<1 \quad \text { and } \quad \frac{\partial v_{i 0}}{\partial n}<0 \quad \text { on } \quad \Gamma, i=1,2
$$

i)

$$
s \in\left(\max \left\{2+1 / 2, \frac{b_{1}^{*}}{\pi-\delta}, \frac{a_{1}^{*}}{\pi-\delta}\right\}, \frac{5 \pi}{2 \pi-\delta}\right) \text { if } \delta \in(0, \pi / 5),
$$

ii)

$$
s \in\left(\max \left\{\frac{2 \pi}{\pi-\delta}, \frac{b_{2}^{*}}{\pi-\delta}, \frac{a_{2}^{*}}{\pi-\delta}\right\}, \frac{5 \pi}{2 \pi-\delta}\right) \text { if } \delta \in(\pi / 5, \pi / 4),
$$


where numbers $b_{j}^{*}$ and $a_{j}^{*}, j=1,2$, are some positive constants depending on initial data (more detailed definitions of these numbers are given in Section 3). Then for some $T$ there exists a unique solution $\left(v_{1}(x, t), v_{2}(x, t), \rho(\omega, t)\right)$ of problem (2.16) for $t \in[0, T]$ such that $v_{i}(x, t) \in E_{s}^{2+\alpha, \alpha, \alpha}\left(\bar{\Omega}_{i T}\right), i=1,2, \rho(\omega, t) \in N_{s, \gamma}^{2+\alpha}$.

It should be remarked that condition (2.20) means that a more viscous fluid is displaced by a less viscous fluid, and then the Muskat problem (2.1)-(2.5) without surface tension is well-posed (see [28] and [1]).

Corollary 2.1. Note that under assumptions of Theorem 2.1 the initial corner point $O$ does not move and the geometry of the initial shape of the free boundary near $O$ is preserved in time. In other words, the results of Theorem 2.1 guarantee the existence of the "waiting time" for angle $\delta \in(0, \pi / 4)$ in the Muskat problem (2.1)-(2.5) in the case of zero surface tension.

\subsection{A perturbation form of system $\left(M_{1}\right)$}

In this subsection, we linearize the system $M_{1}$ on the initial data and rewrite it as a system $\Im \mathbf{z}=F(\mathbf{z})$, where $\Im$ is a linear operator and $F(\mathbf{z})$ is a nonlinear perturbation.

From (2.14) for $t=0$, we have

$$
\begin{gathered}
\frac{\partial \rho}{\partial t}(\omega, 0)=-k_{1}\left(S(\omega, 0,0) \frac{\partial v_{10}}{\partial \lambda}+S_{1}(\omega, 0,0) \frac{\partial v_{10}}{\partial \omega}\right) \\
=-k_{2}\left(S(\omega, 0,0) \frac{\partial v_{20}}{\partial \lambda}+S_{1}(\omega, 0,0) \frac{\partial v_{20}}{\partial \omega}\right) .
\end{gathered}
$$

Let a function $m(\omega, t)$ be such that

$$
m(\omega, 0)=0,\left.\quad \frac{\partial m(\omega, t)}{\partial t}\right|_{t=0}=\frac{\partial \rho}{\partial t}(\omega, 0)
$$

As an example of the function $m(\omega, t)$, we can take $m(\omega, t)=t \frac{\partial \rho}{\partial t}(\omega, 0)$.

We introduce the new unknown functions in the following way:

$$
\begin{gathered}
\sigma(\omega, t)=\rho(\omega, t)-m(\omega, t), \\
\theta_{i}(x, t)=v_{i}(x, t)-v_{i 0}(x)-\left(\nabla_{x} v_{i 0} \cdot \bar{e}_{\sigma}\right),
\end{gathered}
$$

where

$$
\bar{e}_{\sigma}=\frac{\partial x}{\partial \lambda} \chi(\lambda) \sigma(\omega, t), \quad x=\left(x_{1}, x_{2}\right)
$$


Now we rewrite the system $\left(M_{1}\right)$ in terms of the functions $\sigma(\omega, t), \theta_{i}(x, t), i=1,2$, and after some calculations get the problem in the form:

$$
\begin{gathered}
\frac{\partial^{2} \theta_{i}}{\partial x_{1}^{2}}+\frac{\partial^{2} \theta_{i}}{\partial x_{2}^{2}}=F_{0 i}\left(\theta_{i}, \sigma\right) \quad \text { in } \Omega_{i T}, \\
\theta_{1}(x, t)-\theta_{2}(x, t)=\nabla_{x} v_{10} \cdot \bar{e}_{\sigma}-\nabla_{x} v_{20} \cdot \bar{e}_{\sigma} \equiv \sigma A(x) \quad \text { on } \Gamma_{T}, \\
\frac{\partial \sigma}{\partial t}=-k_{1}\left[a(x) \frac{\partial \theta_{1}}{\partial n}+a_{1}(x) \frac{\partial \sigma}{\partial \omega}\right]+F_{1}\left(\theta_{1}, \sigma\right) \quad \text { on } \Gamma_{T}, \\
\frac{\partial \theta_{1}}{\partial n}-k \frac{\partial \theta_{2}}{\partial n}+a_{2}(x) \frac{\partial \sigma}{\partial \omega}=F_{2}\left(\theta_{1}, \theta_{2}, \sigma\right) \quad \text { on } \Gamma_{T}, \\
\left.\frac{\partial \theta_{i}}{\partial x_{1}}\right|_{x_{1}=0}=-\frac{\partial\left(\nabla_{x} v_{i 0} \cdot \bar{e}_{\sigma}\right)}{\partial x_{1}} \equiv F_{3}(\sigma), \theta_{i}(x, t)=0 \quad \text { on } \quad \Gamma_{i T}, \\
\sigma(\omega, 0)=\left.\frac{\partial \sigma(\omega, t)}{\partial t}\right|_{t=0}=0, \quad \theta_{i}(x, 0)=0 .
\end{gathered}
$$

The properties of the functions $A(x), a_{i}(x), a(x) F_{0 i}, i=1,2, F_{1}, F_{2}$ will be described later on.

$\mathrm{R}$ e $\mathrm{m}$ a r k 2.1. Note that $F_{3}(\sigma) \equiv 0$ if we look for the solution in the class of $\sigma \in N_{s, \gamma}^{2+\alpha}$ and take into account definition (2.24) of the vector $\bar{e}_{\sigma}$.

Let

$$
\begin{gathered}
\bar{F}_{1}\left(\theta_{1}, \sigma\right)=F_{1}\left(\theta_{1}, \sigma\right)+k_{1} a_{1}(x) \frac{A_{\omega}(x)}{A(x)} \sigma, \bar{F}_{2}\left(\theta_{1}, \theta_{2}, \sigma\right)=F_{2}\left(\theta_{1}, \theta_{2}, \sigma\right)+a_{2}(x) \frac{A_{\omega}(x)}{A(x)} \sigma, \\
A_{1}(x)=\frac{a_{1}(x)}{A(x)}, \quad A_{2}(x)=\frac{a_{2}(x)}{A(x)} .
\end{gathered}
$$

Then we can rewrite conditions (2.27) and (2.28) as

$$
\begin{gathered}
\frac{\partial \sigma}{\partial t}=-k_{1} a(x) \frac{\partial \theta_{1}}{\partial n}-k_{1} a_{1}(x) \frac{\partial \sigma}{\partial \omega}-k_{1} \frac{a_{1}(x) A_{\omega}(x)}{A(x)} \sigma+\bar{F}_{1}\left(\theta_{1}, \sigma\right) \quad \text { on } \Gamma_{T}, \\
\frac{\partial \theta_{1}}{\partial n}-k \frac{\partial \theta_{2}}{\partial n}+a_{2}(x) \frac{\partial \sigma}{\partial \omega}+a_{2}(x) \frac{A_{\omega}(x)}{A(x)} \sigma=\bar{F}_{2}\left(\theta_{1}, \theta_{2}, \sigma\right) \quad \text { on } \Gamma_{T} .
\end{gathered}
$$

After that we can find the term $\sigma_{\omega}$ from condition (2.26) and substitute it into (2.31), (2.32). Thus we have got the following system $\left(M_{2}\right)$ :

$$
\begin{gathered}
\frac{\partial^{2} \theta_{i}}{\partial x_{1}^{2}}+\frac{\partial^{2} \theta_{i}}{\partial x_{2}^{2}}=F_{0 i}\left(\theta_{i}, \sigma\right) \quad \text { in } \Omega_{i T}, i=1,2 \\
\theta_{1}(x, t)-\theta_{2}(x, t)=A(x) \sigma \quad \text { on } \Gamma_{T}
\end{gathered}
$$




$$
\begin{gathered}
\frac{\partial \sigma}{\partial t}=-k_{1} a(x) \frac{\partial \theta_{1}}{\partial n}-k_{1} A_{1}(x)\left(\frac{\partial \theta_{1}}{\partial \omega}-\frac{\partial \theta_{2}}{\partial \omega}\right)+\bar{F}_{1}\left(\theta_{1}, \sigma\right) \quad \text { on } \Gamma_{T}, \\
\frac{\partial \theta_{1}}{\partial n}-k \frac{\partial \theta_{2}}{\partial n}+A_{2}(x)\left(\frac{\partial \theta_{1}}{\partial \omega}-\frac{\partial \theta_{2}}{\partial \omega}\right)=\bar{F}_{2}\left(\theta_{1}, \theta_{2}, \sigma\right) \quad \text { on } \Gamma_{T}, \\
\left.\frac{\partial \theta_{i}}{\partial x_{1}}\right|_{x_{1}=0}=0, \theta_{i}(x, t)=0 \quad \text { on } \quad \Gamma_{i T}, \\
\sigma(\omega, 0)=\left.\frac{\partial \sigma(\omega, t)}{\partial t}\right|_{t=0}=0, \quad \theta_{i}(x, 0)=0 .
\end{gathered}
$$

Now the system $\left(M_{2}\right)$ can be written as

$$
\Im \mathbf{z}=\mathbf{F}(\mathbf{z}) \text {, where } \mathbf{z}=\left(\theta_{1}, \theta_{2}, \sigma\right) .
$$

Note that if we freeze the functional arguments in the functions $F_{0 i}\left(\theta_{i}, \sigma\right), \bar{F}_{1}\left(\theta_{1}, \sigma\right)$, $\bar{F}_{2}\left(\theta_{1}, \theta_{2}, \sigma\right)$, then system $(2.39)$ or $(2.33)-(2.38)$ will be a linear system with variable coefficients, which will be studied in detail in Section 4 .

To illustrate the system $\left(M_{2}\right)$, we describe it in a vicinity of the angle point $O=(0,0)$ where $\bar{l}(\omega)=(0,-1)$. Let $y_{2}=\varphi\left(y_{1}\right)$ with $\varphi^{\prime}\left(y_{1}\right)>0$ be the equation of $\Gamma=\Gamma(0)$ in the mentioned above vicinity, where $\varphi(0)=0$ and $\varphi^{\prime}(0)=\cot \frac{\delta}{2}$. As a parameter along $\Gamma(0)$, here we take $\omega(y)=y_{1}$, and transformation (2.8) takes the form

$$
y_{1}=x_{1}, y_{2}=x_{2}-\chi(z) \rho\left(x_{1}, t\right), z=x_{2}-\varphi\left(x_{1}\right) .
$$

From (2.40) it follows that

$$
\begin{gathered}
\frac{\partial x_{1}}{\partial y_{1}}=1, \quad \frac{\partial x_{1}}{\partial y_{2}}=0, \\
\frac{\partial x_{2}}{\partial y_{2}}=\frac{1}{1-\chi_{z} \rho}, \quad \frac{\partial x_{2}}{\partial y_{1}}=-\frac{\chi_{z} \rho \varphi_{x_{1}}-\chi \rho_{x_{1}}}{1-\chi_{z} \rho}, \quad \frac{\partial^{2} x_{2}}{\partial y_{2}^{2}}=\frac{\chi_{z z} \rho}{\left(1-\chi_{z} \rho\right)^{3}}, \\
\frac{\partial^{2} x_{2}}{\partial y_{1}^{2}}=\frac{-\chi_{z z}\left(\frac{\partial x_{2}}{\partial y_{1}}-\frac{\partial \varphi}{\partial x_{1}}\right) \frac{\partial \varphi}{\partial x_{1}} \rho-\chi_{z} \rho \frac{\partial^{2} \varphi}{\partial x_{1}^{2}}-\chi_{z} \frac{\partial \rho}{\partial x_{1}} \frac{\partial \varphi}{\partial x_{1}}+\chi_{z}\left(\frac{\partial x_{2}}{\partial y_{1}}-\frac{\partial \varphi}{\partial x_{1}}\right) \frac{\partial \rho}{\partial x_{1}}+\chi \frac{\partial^{2} \rho}{\partial x_{1}^{2}}}{1-\chi_{z} \rho} \\
-\left[\chi_{z z}\left(\frac{\partial x_{2}}{\partial y_{1}}-\frac{\partial \varphi}{\partial x_{1}}\right) \rho+\chi_{z} \frac{\partial \rho}{\partial x_{1}}\right] \frac{\chi_{z} \rho \frac{\partial \varphi}{\partial x_{1}}-\chi_{\frac{\partial \rho}{\partial x_{1}}}}{\left(1-\chi_{z} \rho\right)^{2}} .
\end{gathered}
$$

As before, we set

$$
p_{i}\left(y_{1}, y_{2}, t\right)=p_{i}\left(x_{1}, y_{2}\left(x_{1}, x_{2}, t\right), t\right)=v_{i}\left(x_{1}, x_{2}, t\right),
$$


and equation (2.1) becomes

$$
\begin{gathered}
\frac{\partial^{2} v_{i}}{\partial x_{1}^{2}}+2 \frac{\partial^{2} v_{i}}{\partial x_{1} \partial x_{2}} \frac{\partial x_{2}}{\partial y_{1}}+\frac{\partial^{2} v_{i}}{\partial x_{2}^{2}}\left[\left(\frac{\partial x_{2}}{\partial y_{1}}\right)^{2}+\left(\frac{\partial x_{2}}{\partial y_{2}}\right)^{2}\right] \\
+\frac{\partial v_{i}}{\partial x_{2}}\left[\frac{\partial^{2} x_{2}}{\partial y_{1}^{2}}+\frac{\partial^{2} x_{2}}{\partial y_{2}^{2}}\right]=0 \quad \text { in } \quad \Omega_{i T} .
\end{gathered}
$$

The free boundary $\Gamma(t)$ in Problem $(\mathrm{M})$ has the representation near $O$

$$
\Phi_{\rho}(y, t)=-y_{2}+\varphi\left(y_{1}\right)-\rho\left(y_{1}, t\right)=0
$$

such that

$$
\frac{\partial \Phi_{\rho}}{\partial y_{1}} \frac{d y_{1}}{d t}+\frac{\partial \Phi_{\rho}}{\partial y_{2}} \frac{d y_{2}}{d t}=-\frac{\partial \Phi_{\rho}}{\partial t}, \quad n=\frac{\nabla_{y} \Phi_{\rho}}{\left|\nabla_{y} \Phi_{\rho}\right|}
$$

and hence

$$
V_{n}=\nabla_{y} p_{i} \cdot n=\frac{\rho_{t}}{\left|\nabla_{y} \Phi_{\rho}\right|}
$$

On the other hand,

$$
\frac{\partial p_{i}}{\partial n}=\nabla_{y} p_{i} \cdot n=\frac{\nabla_{y} p_{i}}{\left|\nabla_{y} \Phi_{\rho}\right|} \nabla_{y} \Phi_{\rho}
$$

After using the relation $\nabla_{y} \Phi_{\rho}=\left(\varphi_{x_{1}}-\rho_{x_{1}},-1\right)$, boundary condition (2.3) takes the form

$$
\frac{\partial \rho}{\partial t}=-k_{i}\left[\left(\varphi_{x_{1}}-\rho_{x_{1}}\right)\left(\frac{\partial v_{i}}{\partial x_{1}}-\frac{\partial v_{i}}{\partial x_{2}} \rho_{x_{1}}\right)-\frac{\partial v_{i}}{\partial x_{2}}\right], i=1,2
$$

Since

$$
\frac{\partial v_{i}}{\partial n}=\frac{\partial v_{i}}{\partial x_{1}} \frac{\varphi_{x_{1}}}{\sqrt{1+\varphi_{x_{1}}^{2}}}-\frac{\partial v_{i}}{\partial x_{2}} \frac{1}{\sqrt{1+\varphi_{x_{1}}^{2}}},
$$

another form of the previous equation is $(i=1,2)$,

$$
\frac{\partial \rho}{\partial t}=-k_{i}\left[\frac{\partial v_{i}}{\partial n} \sqrt{1+\varphi_{x_{1}}^{2}}-\frac{\partial \rho}{\partial x_{1}}\left(\frac{\partial v_{i}}{\partial x_{1}}+\left(\varphi_{x_{1}}-\rho_{x_{1}}\right) \frac{\partial v_{i}}{\partial x_{2}}\right)\right] \text { on } \Gamma_{T} .
$$

Boundary conditions (2.2) and (2.4) preserve their forms

$$
\begin{gathered}
v_{1}-v_{2}=0 \quad \Gamma_{T}, \\
v_{i}=\psi_{i}(x) \quad \text { on } \quad \Gamma_{i T},
\end{gathered}
$$

and initial conditions are

$$
v_{i}(x, 0)=v_{i 0}(x), \quad \rho\left(x_{1}, 0\right)=0 .
$$


In this simple case, the nonlinear problem is described by equations (2.41), (2.43)(2.46).

Since $\rho\left(x_{1}, 0\right)=0$ from $(2.43)$, we get

$$
\left.\frac{\partial \rho}{\partial t}\right|_{t=0}=-k_{1} \frac{\partial v_{10}}{\partial n} \sqrt{1+\varphi_{x_{1}}^{2}} .
$$

Let

$$
m\left(x_{1}, t\right)=-t k_{1} \frac{\partial v_{10}}{\partial n} \sqrt{1+\varphi_{x_{1}}^{2}} \equiv t m_{0}(x),
$$

and

$$
\rho\left(x_{1}, t\right)=\sigma\left(x_{1}, t\right)+m\left(x_{1}, t\right)
$$

such that

$$
\sigma\left(x_{1}, 0\right)=0,\left.\quad \frac{\partial \sigma}{\partial t}\right|_{t=0}=0 .
$$

Introduce the function

$$
w_{i}(x, t)=v_{i}(x, t)-v_{i 0}(x), \quad w_{i}(x, 0)=0 .
$$

Next we rewrite equations (2.41), (2.43)-(2.46) in the terms of $w_{i}(x, t), \sigma\left(x_{1}, t\right)$. Equation (2.41) is transformed to

$$
\frac{\partial^{2} w_{i}}{\partial x_{1}^{2}}+\frac{\partial^{2} w_{i}}{\partial x_{2}^{2}}+\chi(z) \frac{\partial v_{i 0}}{\partial x_{2}} \frac{\partial^{2} \sigma}{\partial x_{1}^{2}}=g_{0 i}\left(w_{i}, \sigma\right) .
$$

Below we will define the function $g_{0 i}\left(w_{i}, \sigma\right)$. The form of the left-hand side here suggests another change of unknown functions

$$
\theta_{i}(x, t)=w_{i}(x, t)+\chi(z) \frac{\partial v_{i 0}}{\partial x_{2}} \sigma\left(x_{1}, t\right) .
$$

This step explains the appearance of the last term in the right-hand side of equation (2.23).

Finally we have

$$
\Delta \theta_{i}=F_{0 i}\left(\theta_{i}, \sigma\right) \text { in } \Omega_{i T}, i=1,2,
$$

where

$$
\begin{gathered}
F_{0 i}\left(\theta_{i}, \sigma\right) \equiv g_{0 i}\left(\theta_{i}, \sigma\right)-\left(\chi \frac{\partial v_{i 0}}{\partial x_{2}}\right)_{x_{1} x_{1}} \sigma-2\left(\chi \frac{\partial v_{i 0}}{\partial x_{2}}\right)_{x_{1}} \sigma_{x_{1}}-\left(\chi \frac{\partial v_{i 0}}{\partial x_{2}}\right)_{x_{2} x_{2}} \sigma \\
g_{0 i}\left(w_{i}, \sigma\right)=-\frac{\partial^{2} v_{i 0}}{\partial x_{1}^{2}}-2 \frac{\partial^{2}\left(v_{i 0}+w_{i}\right)}{\partial x_{1} \partial x_{2}} \frac{\partial x_{2}}{\partial y_{1}}-\frac{\partial^{2} v_{i 0}}{\partial x_{2}^{2}}\left[\left(\frac{\partial x_{2}}{\partial y_{1}}\right)^{2}+\left(\frac{\partial x_{2}}{\partial y_{2}}\right)^{2}\right] \\
-\frac{\partial^{2} w_{i}}{\partial x_{2}^{2}}\left[-1+\left(\frac{\partial x_{2}}{\partial y_{1}}\right)^{2}+\left(\frac{\partial x_{2}}{\partial y_{2}}\right)^{2}\right]-\frac{\partial\left(v_{i 0}+w_{i}\right)}{\partial x_{2}}\left(\frac{\partial^{2} x_{2}}{\partial y_{1}^{2}}+\frac{\partial^{2} x_{2}}{\partial y_{2}^{2}}\right)+\chi \frac{\partial v_{i 0}}{\partial x_{2}} \frac{\partial^{2} \sigma}{\partial x_{1}^{2}} .
\end{gathered}
$$


$\mathrm{R}$ e $\mathrm{m}$ a $\mathrm{r} \mathrm{k}$ 2.2. The function $F_{0 i}\left(\theta_{i}, \sigma\right)$ contains the higher derivatives of $\theta_{i}(x, t)$ and $\sigma\left(x_{1}, t\right)$ with coefficients that tend to zero as $t \rightarrow 0$, the "quadratic" terms with respect to $\theta_{i}(x, t)$ and $\sigma\left(x_{1}, t\right)$, and their derivatives, and the terms of minor differential orders of unknown functions.

For example, the factor $\left[-1+\left(\frac{\partial x_{2}}{\partial y_{1}}\right)^{2}+\left(\frac{\partial x_{2}}{\partial y_{2}}\right)^{2}\right]$ tends to zero as $t \rightarrow 0$ by the definition of $\frac{\partial x_{2}}{\partial y_{1}}, \frac{\partial x_{2}}{\partial y_{2}}$, and the equation $\rho(x, 0)=0$. It is easy to check that the coefficient under $\sigma_{x_{1} x_{1}}$ in $F_{0 i}\left(\theta_{i}, \sigma\right)$ is

$$
\chi(z) \frac{\partial v_{i 0}}{\partial x_{2}}\left(\frac{1}{1-\chi_{z}(m+\sigma)}-1\right)=\frac{\chi(z) \chi_{z}(m+\sigma) \frac{\partial v_{i 0}}{\partial x_{2}}}{1-\chi_{z}(m+\sigma)}
$$

and vanishes as $t \rightarrow 0$ due to $\left(m\left(x_{1}, t\right)+\sigma\left(x_{1}, t\right)\right) \rightarrow 0$ as $t \rightarrow 0$.

Since $w_{1}(x, t)=w_{2}(x, t)$ and $\chi(z)=1$ on $\Gamma_{T}$, condition (2.2) takes the form

$$
\theta_{1}(x, t)-\theta_{2}(x, t)=\left(\frac{\partial v_{10}}{\partial x_{2}}-\frac{\partial v_{20}}{\partial x_{2}}\right) \sigma .
$$

By simple calculations, we transform equation (2.43) into

$$
\frac{\partial \sigma}{\partial t}=-k_{i}\left[\frac{\partial \theta_{i}}{\partial n} \sqrt{1+\varphi_{x_{1}}^{2}}-\frac{\partial \sigma}{\partial x_{1}} \frac{\partial v_{i 0}}{\partial x_{1}}+g_{i}\right]
$$

where

$$
\begin{aligned}
& g_{i}\left(\theta_{i}, \sigma\right)=-\sigma \frac{\partial^{2} v_{i 0}}{\partial n \partial x_{2}} \sqrt{1+\varphi_{x_{1}}^{2}}+\sqrt{1+\varphi_{x_{1}}^{2}} \frac{\partial v_{i 0}}{\partial n}+\frac{\partial \sigma}{\partial x_{1}} \frac{\partial v_{i 0}}{\partial x_{1}}+\left(\frac{\partial \sigma}{\partial x_{1}}+\frac{\partial m}{\partial x_{1}}\right) \\
& \times\left[\frac{\partial^{2} v_{i 0}}{\partial x_{2} \partial x_{1}} \sigma-\frac{\partial\left(\theta_{i}+v_{i 0}\right)}{\partial x_{1}}+\frac{\partial v_{i 0}}{\partial x_{2}} \sigma_{x_{1}}\right]-\left(\varphi_{x_{1}}-\sigma_{x_{1}}-m_{x_{1}}\right) \\
& \times\left(\frac{\partial\left(\theta_{i}+v_{i 0}\right)}{\partial x_{2}}-\frac{\partial^{2} v_{i 0}}{\partial x_{2}^{2}} \sigma\right)\left(\frac{\partial \sigma}{\partial x_{1}}+\frac{\partial m}{\partial x_{1}}\right) .
\end{aligned}
$$

$\mathrm{R}$ e $\mathrm{m}$ a $\mathrm{r} \mathrm{k}$ 2.3. $g_{i}\left(\theta_{i}, \sigma\right)$ satisfies to the same properties as $F_{0 i}\left(\theta_{i}, \sigma\right)$, i.e., $g_{i}\left(\theta_{i}, \sigma\right)$ contains the minor terms, the "quadratic" terms and the higher terms with small coefficients as $t \rightarrow 0$.

In reality, (2.54) contains two conditions. The first of them is

$$
\frac{\partial \sigma}{\partial t}=-k_{1}\left(\frac{\partial \theta_{1}}{\partial n} \sqrt{1+\varphi_{x_{1}}^{2}}-\frac{\partial \sigma}{\partial x_{1}} \frac{\partial v_{10}}{\partial x_{1}}\right)+F_{1}\left(\theta_{1}, \sigma\right), F_{1}\left(\theta_{1}, \sigma\right)=-k_{1} g_{1}\left(\theta_{1}, \sigma\right),
$$


and the second one is

$$
\begin{gathered}
\frac{\partial \theta_{1}}{\partial n}-k \frac{\partial \theta_{2}}{\partial n}-\frac{1}{\sqrt{1+\varphi_{x_{1}}^{2}}} \frac{\partial \sigma}{\partial x_{1}}\left(\frac{\partial v_{10}}{\partial x_{1}}-k \frac{\partial v_{20}}{\partial x_{1}}\right)=\frac{1}{\sqrt{1+\varphi_{x_{1}}^{2}}}\left(k g_{2}-g_{1}\right) \\
=F_{2}\left(\theta_{1}, \theta_{2}, \sigma\right) .
\end{gathered}
$$

Equations (2.51), (2.53), (2.55), and (2.56) correspond to (2.25)-(2.28).

We will use the equalities

$$
\begin{gathered}
\frac{\partial \sigma}{\partial x_{1}}=\sqrt{1+\varphi_{x_{1}}^{2}} \frac{\partial \sigma}{\partial \tau} \\
\frac{\partial v_{10}}{\partial x_{2}}-\frac{\partial v_{20}}{\partial x_{2}}=\frac{1-k}{k} \frac{1}{\sqrt{1+\varphi_{x_{1}}^{2}}} \frac{\partial v_{10}}{\partial n} \\
\frac{\partial v_{10}}{\partial x_{1}}-k \frac{\partial v_{20}}{\partial x_{1}}=\frac{1-k}{\sqrt{1+\varphi_{x_{1}}^{2}}} \frac{\partial v_{10}}{\partial \tau}
\end{gathered}
$$

where $\tau$ is a tangent vector to $\Gamma$, and the two last equalities follow from (2.17).

Summing our calculations, we get the next problem. It is necessary to find the functions $\theta_{i}(x, t), i=1,2, \sigma(\tau, t)$ by the conditions

$$
\begin{gathered}
\frac{\partial^{2} \theta_{i}}{\partial x_{1}^{2}}+\frac{\partial^{2} \theta_{i}}{\partial x_{2}^{2}}=F_{0 i}\left(\theta_{i}, \sigma\right) \text { in } \Omega_{i T} \\
\theta_{1}(x, t)-\theta_{2}(x, t)=\frac{1-k}{k} \frac{1}{\sqrt{1+\varphi_{x_{1}}^{2}}} \frac{\partial v_{10}}{\partial n} \sigma \text { on } \Gamma_{T} \\
\frac{\partial \theta_{1}}{\partial n}-k \frac{\partial \theta_{2}}{\partial n}-\frac{(1-k)}{\sqrt{1+\varphi_{x_{1}}^{2}}} \frac{\partial \sigma}{\partial \tau} \frac{\partial v_{10}}{\partial \tau}=F_{2}\left(\theta_{1}, \theta_{2}, \sigma\right) \text { on } \Gamma_{T}, \\
\frac{\partial \sigma}{\partial t}=-k_{1} \sqrt{1+\varphi_{x_{1}}^{2}}\left(\frac{\partial \theta_{1}}{\partial n}-\frac{\partial \sigma}{\partial \tau} \frac{\partial v_{10}}{\partial x_{1}}\right)+F_{1}\left(\theta_{1}, \sigma\right) \text { on } \Gamma_{T}, \\
\theta_{i}(x, t)=0 \text { on } \Gamma_{i T},\left.\frac{\partial \theta_{i}}{\partial x_{1}}\right|_{x_{1}=0}=-\left.\frac{\partial}{\partial x_{1}}\left(\chi\left[x_{2}-\varphi_{x_{1}}\right] \sigma(\omega, t) \frac{\partial v_{i 0}}{\partial x_{2}}\right)\right|_{x_{1}=0} \equiv F_{3}(x, t, \sigma), \\
\sigma(\tau, 0)=0,\left.\frac{\partial \sigma(\tau, t)}{\partial t}\right|_{t=0}=0, \theta_{i}(x, 0)=0,
\end{gathered}
$$

where the functions $F_{0 i}\left(\theta_{i}, \sigma\right), F_{1}\left(\theta_{1}, \sigma\right), F_{2}\left(\theta_{1}, \theta_{2}, \sigma\right)$ are defined in (2.54), (2.55) and (2.56), respectively, and as it follows from Remark $2.1, F_{3}(x, t, \sigma) \equiv 0$ for every $(x, t) \in \Gamma_{T}$.

Our next step is to get rid of $\frac{\partial \sigma}{\partial \tau}$ in the left-hand sides of equations (2.59) and (2.60). To this end, we introduce the functions

$$
\bar{F}_{1}\left(\theta_{1}, \sigma\right)=\frac{F_{1}\left(\theta_{1}, \sigma\right)}{\sqrt{1+\varphi_{x_{1}}^{2}}}-k_{1} \frac{\partial}{\partial \tau}\left(\frac{\partial v_{10}}{\partial n}\left(1+\varphi_{x_{1}}^{2}\right)^{-1 / 2}\right)\left(\varphi_{x_{1}}+\frac{\frac{\partial v_{10}}{\partial \tau}}{\frac{\partial v_{10}}{\partial n}}\right) \sigma,
$$




$$
\bar{F}_{2}\left(\theta_{1}, \theta_{2}, \sigma\right)=F_{2}\left(\theta_{1}, \theta_{2}, \sigma\right)-(1-k) \frac{\partial}{\partial \tau}\left(\frac{\partial v_{10}}{\partial n}\left(1+\varphi_{x_{1}}^{2}\right)^{-1 / 2}\right) \frac{\frac{\partial v_{10}}{\partial \tau}}{\frac{\partial v_{10}}{\partial n}} \sigma .
$$

Then conditions (2.59) and (2.60) can be rewritten as

$$
\begin{gathered}
\frac{\partial \theta_{1}}{\partial n}-k \frac{\partial \theta_{2}}{\partial n}-\frac{(1-k)}{\sqrt{1+\varphi_{x_{1}}^{2}}} \frac{\partial \sigma}{\partial \tau} \frac{\partial v_{10}}{\partial \tau}-(1-k) \frac{\partial}{\partial \tau}\left(\frac{\partial v_{10}}{\partial n}\left(1+\varphi_{x_{1}}^{2}\right)^{-1 / 2}\right) \frac{\frac{\partial v_{10}}{\partial \tau}}{\frac{\partial v_{10}}{\partial n}} \sigma \\
=\bar{F}_{2}\left(\theta_{1}, \theta_{2}, \sigma\right) \text { on } \Gamma_{T} \\
\frac{1}{\sqrt{1+\varphi_{x_{1}}^{2}}} \frac{\partial \sigma}{\partial t}=-k_{1} \frac{\partial \theta_{1}}{\partial n}+k_{1} \frac{\partial \sigma}{\partial \tau}\left(\frac{1}{\sqrt{1+\varphi_{x_{1}}^{2}}} \frac{\partial v_{10}}{\partial \tau}+\frac{\varphi_{x_{1}}}{\sqrt{1+\varphi_{x_{1}}^{2}}} \frac{\partial v_{10}}{\partial n}\right) \\
+k_{1} \frac{\partial}{\partial \tau}\left(\frac{\partial v_{10}}{\partial n}\left(1+\varphi_{x_{1}}^{2}\right)^{-1 / 2}\right)\left(\varphi_{x_{1}}+\frac{\frac{\partial v_{10}}{\partial \tau}}{\frac{\partial v_{10}}{\partial n}}\right) \sigma+\bar{F}_{1}\left(\theta_{1}, \sigma\right) \text { on } \Gamma_{T} .
\end{gathered}
$$

From (2.58), we get

$$
\frac{\partial \sigma}{\partial \tau}=\frac{k \sqrt{1+\varphi_{x_{1}}^{2}}}{(1-k) \frac{\partial v_{10}}{\partial n}}\left(\frac{\partial \theta_{1}}{\partial \tau}-\frac{\partial \theta_{2}}{\partial \tau}\right)-\frac{\sqrt{1+\varphi_{x_{1}}^{2}}}{\frac{\partial v_{10}}{\partial n}} \frac{\partial}{\partial \tau}\left(\frac{\partial v_{10}}{\partial n}\left(1+\varphi_{x_{1}}^{2}\right)^{-1 / 2}\right) \sigma
$$

Substituting this expression for $\frac{\partial \sigma}{\partial \tau}$ into (2.63) and (2.64), we get

$$
\begin{gathered}
\frac{\partial \theta_{1}}{\partial n}-k \frac{\partial \theta_{2}}{\partial n}-k \frac{\frac{\partial v_{10}}{\partial \tau}}{\frac{\partial v_{10}}{\partial n}}\left(\frac{\partial \theta_{1}}{\partial \tau}-\frac{\partial \theta_{2}}{\partial \tau}\right)=\bar{F}_{2}\left(\theta_{1}, \theta_{2}, \sigma\right) \text { on } \Gamma_{T} \\
\frac{1}{\sqrt{1+\varphi_{x_{1}}^{2}}} \frac{\partial \sigma}{\partial t}=-k_{1} \frac{\partial \theta_{1}}{\partial n}+\frac{k_{2}}{1-k}\left(\varphi_{x_{1}}+\frac{\frac{\partial v_{10}}{\partial \tau}}{\frac{\partial v_{10}}{\partial n}}\right)\left(\frac{\partial \theta_{1}}{\partial \tau}-\frac{\partial \theta_{2}}{\partial \tau}\right)+\bar{F}_{1}\left(\theta_{1}, \sigma\right) \text { on } \Gamma_{T} .
\end{gathered}
$$

Now we can find the term $\frac{k_{2}}{1-k} \frac{\frac{\partial v_{10}}{\partial \tau}}{\frac{\partial \tau}{\partial n}}\left(\frac{\partial \theta_{1}}{\partial \tau}-\frac{\partial \theta_{2}}{\partial \tau}\right)$ from (2.65) and substitute it into condition (2.66). As a result, we have

$$
\frac{1-k}{k_{2} \sqrt{1+\varphi_{x_{1}}^{2}}} \frac{\partial \sigma}{\partial t}=\frac{\partial \theta_{1}}{\partial n}-\frac{\partial \theta_{2}}{\partial n}+\varphi_{x_{1}}\left(\frac{\partial \theta_{1}}{\partial \tau}-\frac{\partial \theta_{2}}{\partial \tau}\right)+\frac{1-k}{k_{2}} \bar{F}_{1}\left(\theta_{1}, \sigma\right)-\frac{\bar{F}_{2}}{k} \text { on } \Gamma_{T} \text {. }
$$

Thus system (2.57)-(2.62) can be represented as

$$
\begin{gathered}
\frac{\partial^{2} \theta_{i}}{\partial x_{1}^{2}}+\frac{\partial^{2} \theta_{i}}{\partial x_{2}^{2}}=F_{0 i}\left(\theta_{i}, \sigma\right) \text { in } \Omega_{i T}, \\
\theta_{1}(x, t)-\theta_{2}(x, t)=\frac{1-k}{k} \frac{1}{\sqrt{1+\varphi_{x_{1}}^{2}}} \frac{\partial v_{10}}{\partial n} \sigma \text { on } \Gamma_{T},
\end{gathered}
$$




$$
\begin{gathered}
\frac{\partial \theta_{1}}{\partial n}-k \frac{\partial \theta_{2}}{\partial n}-k \frac{\frac{\partial v_{10}}{\partial \tau}}{\frac{\partial v_{10}}{\partial n}}\left(\frac{\partial \theta_{1}}{\partial \tau}-\frac{\partial \theta_{2}}{\partial \tau}\right)=\bar{F}_{2}\left(\theta_{1}, \theta_{2}, \sigma\right) \text { on } \Gamma_{T} \\
\frac{1-k}{k_{2} \sqrt{1+\varphi_{x_{1}}^{2}}} \frac{\partial \sigma}{\partial t}=\frac{\partial \theta_{1}}{\partial n}-\frac{\partial \theta_{2}}{\partial n}+\varphi_{x_{1}}\left(\frac{\partial \theta_{1}}{\partial \tau}-\frac{\partial \theta_{2}}{\partial \tau}\right)+\frac{1-k}{k_{2}} \bar{F}_{1}\left(\theta_{1}, \sigma\right)-\frac{\bar{F}_{2}}{k} \text { on } \Gamma_{T} \\
\theta_{i}(x, t)=0 \text { on } \Gamma_{i T},\left.\frac{\partial \theta_{i}}{\partial x_{1}}\right|_{x_{1}=0}=0 \\
\sigma(\tau, 0)=0,\left.\frac{\partial \sigma(\tau, t)}{\partial t}\right|_{t=0}=0, \theta_{i}(x, 0)=0
\end{gathered}
$$

$\mathrm{R}$ e $\mathrm{m}$ a $\mathrm{r} \mathrm{k}$ 2.4. As it follows from Remarks 2.2 and 2.3, the functions $F_{0 i}\left(\theta_{i}, \sigma\right), \bar{F}_{1}\left(\theta_{1}, \sigma\right)$ and $\bar{F}_{2}\left(\theta_{1}, \theta_{2}, \sigma\right)$ in a vicinity of the angle point $O$ contain the higher derivatives of $\theta_{i}(x, t)$ and $\sigma\left(x_{1}, t\right)$ with coefficients that tend to zero as $t \rightarrow 0$, the "quadratic" terms with respect to $\theta_{i}(x, t)$ and $\sigma\left(x_{1}, t\right)$, and their derivatives, and the terms of minor differential orders of unknown functions. Moreover, the same results are preserved outside the angle point $O$.

In the sequel we need a somewhat different form of system (2.67)-(2.72). It deals with the view of condition (2.70) which can be modified if we look for $\sigma_{t}$ from condition (2.68) and after that substitute this term into (2.70). Thus, after some simple calculations we have the system

$$
\begin{aligned}
& \frac{\partial^{2} \theta_{i}}{\partial x_{1}^{2}}+\frac{\partial^{2} \theta_{i}}{\partial x_{2}^{2}}=F_{0 i}\left(\theta_{i}, \sigma\right) \text { in } \Omega_{i T}, \\
& \theta_{1}(x, t)-\theta_{2}(x, t)=\frac{1-k}{k} \frac{1}{\sqrt{1+\varphi_{x_{1}}^{2}}} \frac{\partial v_{10}}{\partial n} \sigma \text { on } \Gamma_{T}, \\
& \frac{\partial \theta_{1}}{\partial n}-k \frac{\partial \theta_{2}}{\partial n}-k \frac{\frac{\partial v_{10}}{\partial \tau}}{\frac{\partial v_{10}}{\partial n}}\left(\frac{\partial \theta_{1}}{\partial \tau}-\frac{\partial \theta_{2}}{\partial \tau}\right)=\bar{F}_{2}\left(\theta_{1}, \theta_{2}, \sigma\right) \text { on } \Gamma_{T}, \\
& \frac{1}{k_{1} \frac{\partial v_{10}}{\partial n}}\left(\frac{\partial \theta_{1}}{\partial t}-\frac{\partial \theta_{2}}{\partial t}\right)-\left(\frac{\partial \theta_{1}}{\partial n}-\frac{\partial \theta_{2}}{\partial n}\right)-\varphi_{x_{1}}\left(\frac{\partial \theta_{1}}{\partial \tau}-\frac{\partial \theta_{2}}{\partial \tau}\right) \\
& =\frac{1-k}{k_{2}} \bar{F}_{1}\left(\theta_{1}, \sigma\right)-\frac{\bar{F}_{2}}{k} \equiv \overline{\bar{F}}_{1}\left(\theta_{1}, \theta_{2}, \sigma\right) \text { on } \Gamma_{T} \\
& \theta_{i}(x, t)=0 \text { on } \Gamma_{i T},\left.\frac{\partial \theta_{i}}{\partial x_{1}}\right|_{x_{1}=0}=0 \\
& \sigma(\tau, 0)=0,\left.\frac{\partial \sigma(\tau, t)}{\partial t}\right|_{t=0}=0, \theta_{i}(x, 0)=0 .
\end{aligned}
$$




\section{Model Problems}

As is known, to construct a model problem near the boundary by using the Schauder method, it is necessary to fix the coefficients of the original problem at the boundary point and, if necessary, straighten the boundary in some vicinity of the fixed point.

\subsection{A model problem near a corner point}

Let now the fixed point be the angle point in problem (2.67)-(2.72). Denote

$$
\begin{gathered}
G_{1}=\left\{\left(x_{1}, x_{2}\right): x_{1}>0,-\infty<x_{2}<x_{1} \cot \frac{\delta}{2}\right\}, \quad G_{1 T}=G_{1} \times[0, T], \\
G_{2}=\left\{\left(x_{1}, x_{2}\right): x_{1}>0, x_{1} \cot \frac{\delta}{2}<x_{2}<\infty\right\}, \quad G_{2 T}=G_{2} \times[0, T], \\
g=\left\{\left(x_{1}, x_{2}\right): x_{1}>0, x_{2}=x_{1} \cot \frac{\delta}{2}\right\}, \quad g_{T}=g \times[0, T] .
\end{gathered}
$$

After some evident transformations made to eliminate the unknown function $\sigma(\omega, t)$ (see $(2.73)-(2.78)$ ), we obtain the model problem in a plane corner for the functions $u_{1}(x, t), u_{2}(x, t)$ :

$$
\begin{gathered}
\frac{\partial^{2} u_{i}}{\partial x_{1}^{2}}+\frac{\partial^{2} u_{i}}{\partial x_{2}^{2}}=0 \text { in } G_{i T}, u_{i}(x, 0)=0, i=1,2 \\
r^{-1-\gamma}\left(\frac{\partial u_{1}}{\partial t}-\frac{\partial u_{2}}{\partial t}\right)+\left(\frac{\partial u_{1}}{\partial n}-\frac{\partial u_{2}}{\partial n}\right)+h\left(\frac{\partial u_{1}}{\partial r}-\frac{\partial u_{2}}{\partial r}\right)=f(r, t) \text { on } g_{T}, \\
\frac{\partial u_{1}}{\partial n}-k \frac{\partial u_{2}}{\partial n}-k d_{1}\left(\frac{\partial u_{1}}{\partial r}-\frac{\partial u_{2}}{\partial r}\right)=0 \text { on } g_{T} \\
\frac{\partial u_{1}}{\partial n}=0 \text { on }\left\{x_{1}=0, x_{2}<0\right\} \times[0, T] ; \quad \frac{\partial u_{2}}{\partial n}=0 \text { on }\left\{x_{1}=0, x_{2}>0\right\} \times[0, T]
\end{gathered}
$$

where $r=\sqrt{x_{1}^{2}+x_{2}^{2}}, 0<k<1, h=\cot \frac{\delta}{2}, \delta \in(0, \pi), d_{1}=\left.\frac{\partial v_{01} / d r}{\partial v_{01} / d n}\right|_{x_{1}=x_{2}=0}$, and $\gamma$ is some positive number defined by (2.19). We took into account the asymptotic behaviour of the function $\frac{\partial v_{01}}{\partial n}$ as $x \rightarrow 0$ in problem (2.17), $\frac{\partial v_{01}}{\partial n} \sim A_{3} r^{1+\gamma}$, $A_{3}$ being a negative constant, and then assumed without loss of generality that $\left(-k_{1} A_{3}\right)=1$.

Note that problem (3.1)-(3.4) was studied in the recent paper [9] (see Section 3 there), where the one-valued solvability of this problem was proved under more general assumption on the constants. In our case, we reformulate the results of Theorems 3.1 and 3.2 and Remark 4.1 from[9] as:

Theorem 3.1. Let $\alpha \in(0,1)$ and

$$
f(x, t) \in E_{s-1}^{1+\alpha, \alpha, \alpha}\left(\bar{g}_{T}\right), f(x, t)=0 \text { if either } t<0 \text { or }|x|>R_{0}
$$

for some positive $R_{0}$, and 
i)

$$
s \in\left(\max \left\{2+1 / 2, \frac{b_{1}^{*}}{\pi-\delta}, \frac{a_{1}^{*}}{\pi-\delta}\right\}, \frac{5 \pi}{2 \pi-\delta}\right) \text { if } \delta \in(0, \pi / 5),
$$

ii)

$$
s \in\left(\max \left\{\frac{2 \pi}{\pi-\delta}, \frac{b_{2}^{*}}{\pi-\delta}, \frac{a_{2}^{*}}{\pi-\delta}\right\}, \frac{5 \pi}{2 \pi-\delta}\right) \text { if } \delta \in(\pi / 5, \pi / 4),
$$

where the numbers $b_{j}^{*}$ and $a_{j}^{*}, j=1,2$, are some positive constants depending on $k, h, d_{1}$, and $\delta$. Then there exists a unique solution $\left(u_{1}, u_{2}\right)$ of problem (3.1)-(3.4) such that $u_{i} \in E_{s}^{2+\alpha, \alpha, \alpha}\left(\bar{G}_{i T}\right)$ and the estimates hold

$$
\begin{gathered}
\sum_{i=1}^{2}\left\{\left\|u_{i}\right\|_{E_{s}^{2+\alpha, \alpha, \alpha}\left(\bar{G}_{i T}\right)}+\left\|r^{-1-\gamma} \partial u_{i} / \partial t\right\|_{E_{s-1}^{1+\alpha, \alpha, \alpha}\left(\bar{g}_{T}\right)}\right\} \leq \text { const }\|f\|_{E_{s-1}^{1+\alpha, \alpha, \alpha}\left(\bar{g}_{T}\right)}, \\
\sum_{i=1}^{2}\left\|u_{i}\right\|_{E_{s}^{1+\alpha, \alpha, \alpha}\left(\bar{G}_{i T}\right)} \leq \operatorname{const} T^{\alpha^{*}-\alpha} \max \left\{1, R_{0}^{\alpha^{*} \gamma}\right\}\|f\|_{E_{s-1}^{1+\alpha, \alpha, \alpha}\left(\bar{g}_{T}\right)}
\end{gathered}
$$

where $\alpha<\alpha^{*}<1$.

As it is shown in [9] (see Theorem 2.1 and Remark 4.1 there), the results of Theorem 3.1 are preserved in the case of nonhomogeneous boundary value problem corresponding to (3.1)-(3.4),

$$
\begin{gathered}
\frac{\partial^{2} u_{i}}{\partial x_{1}^{2}}+\frac{\partial^{2} u_{i}}{\partial x_{2}^{2}}=f_{0 i} \text { in } G_{i T}, u_{i}(x, 0)=0, i=1,2 \\
r^{-1-\gamma}\left(\frac{\partial u_{1}}{\partial t}-\frac{\partial u_{2}}{\partial t}\right)+\left(\frac{\partial u_{1}}{\partial n}-\frac{\partial u_{2}}{\partial n}\right)+h\left(\frac{\partial u_{1}}{\partial r}-\frac{\partial u_{2}}{\partial r}\right)=f(r, t) \text { on } g_{T}, \\
\frac{\partial u_{1}}{\partial n}-k \frac{\partial u_{2}}{\partial n}-k d_{1}\left(\frac{\partial u_{1}}{\partial r}-\frac{\partial u_{2}}{\partial r}\right)=f_{1} \text { on } g_{T} \\
\frac{\partial u_{1}}{\partial n}=0 \text { on }\left\{x_{1}=0, x_{2}<0\right\} \times[0, T] ; \quad \frac{\partial u_{2}}{\partial n}=0 \text { on }\left\{x_{1}=0, x_{2}>0\right\} \times[0, T]
\end{gathered}
$$

For the sake of convenience, we reformulate this results as follows.

Theorem 3.2. Let $s$ satisfy conditions $i$ ), ii) from Theorem 3.1, $f_{0 i} \in$ $E_{s-2}^{\alpha, \alpha, \alpha}\left(\bar{G}_{i T}\right), i=1,2, f_{1}, f \in E_{s-1}^{1+\alpha, \alpha, \alpha}\left(\bar{g}_{T}\right)$, and $f_{0 i}, f_{1}, f=0$ if either $t \leq 0$ or $|x|>R_{0}$, for some positive number $R_{0}$. Then there exists a unique solution $u_{i} \in E_{s}^{2+\alpha, \alpha, \alpha}\left(\bar{G}_{i T}\right), i=1,2$, of problem (3.7)-(3.10) and the estimate holds

$$
\sum_{i=1}^{2}\left\|u_{i}\right\|_{E_{s}^{2+\alpha, \alpha, \alpha}\left(\bar{G}_{i T}\right)}+\left\|r^{-1-\gamma}\left(\partial u_{1} / \partial t-\partial u_{2} / \partial t\right)\right\|_{E_{s-1}^{1+\alpha, \alpha, \alpha}\left(\bar{g}_{T}\right)}
$$




$$
\leq \operatorname{const}\left[\sum_{i=1}^{2}\left\|f_{0 i}\right\|_{E_{s-2}^{\alpha, \alpha, \alpha}\left(\bar{G}_{i T}\right)}+\left\|f_{1}\right\|_{E_{s-1}^{1+\alpha, \alpha, \alpha}\left(\bar{g}_{T}\right)}+\|f\|_{E_{s-1}^{1+\alpha, \alpha, \alpha}\left(\bar{g}_{T}\right)}\right] .
$$

\subsection{A model problem near a smooth part of the interface}

We consider now the case when the fixed point $x=\tilde{x}$ is outward to the $\varepsilon_{0^{-}}$ vicinity of the corner point $O$. Then some evident transformations in the system $\left(M_{2}\right)$ of equations $(2.33)-(2.38)$ lead to the following problem in half-spaces. Let

$$
\begin{array}{cl}
R_{+}^{2}=\left\{\left(x_{1}, x_{2}\right): x_{1} \in R^{1}, x_{2}>0\right\}, & R_{+T}^{2}=R_{+}^{2} \times(0, T) ; \\
R_{-}^{2}=\left\{\left(x_{1}, x_{2}\right): x_{1} \in R^{1}, x_{2}<0\right\}, & R_{-T}^{2}=R_{-}^{2} \times(0, T) ; \\
R_{ \pm T}^{2}=R_{ \pm}^{2} \times(0, T) ; \quad R_{T}^{1}=R^{1} \times(0, T) ; & a_{0}=-\left.\left(k_{1} \frac{\partial v_{01}}{\partial n}\right)^{-1}\right|_{x=\tilde{x}} .
\end{array}
$$

We search a solution $\left(u_{+}\left(x_{1}, x_{2}, t\right), u_{-}\left(x_{1}, x_{2}, t\right)\right)$ bounded at the infinity by the conditions

$$
\begin{gathered}
\Delta_{x} u_{ \pm}=f_{0}^{ \pm} \quad \text { in } \quad R_{ \pm T}^{2} ; \quad u_{ \pm}\left(x_{1}, x_{2}, 0\right)=0, \quad x \in R_{ \pm}^{2} \\
a_{0}\left(\frac{\partial u_{-}}{\partial t}-\frac{\partial u_{+}}{\partial t}\right)+\left(\frac{\partial u_{-}}{\partial n}-\frac{\partial u_{+}}{\partial n}\right)+a_{1}\left(\frac{\partial u_{-}}{\partial x_{1}}-\frac{\partial u_{+}}{\partial x_{1}}\right)=f_{1}, \quad(x, t) \in \overline{R_{T}^{1}} \\
\frac{\partial u_{-}}{\partial n}-k \frac{\partial u_{+}}{\partial n}-k a_{2}\left(\frac{\partial u_{-}}{\partial x_{1}}-\frac{\partial u_{+}}{\partial x_{1}}\right)=f_{2}, \quad(x, t) \in \overline{R_{T}^{1}}
\end{gathered}
$$

where $\Delta_{x}$ is the Laplace operator with respect to $\left(x_{1}, x_{2}\right) ; n$ is the normal to $R^{1}$ directed in $R_{-}^{2} ; a_{i}, i=0,1,2$, are some given constants, $a_{1} \geq 0 ; f_{0}^{ \pm}, f_{i}, i=1,2$, are the given functions such that

$$
f_{0}^{ \pm}, f_{1}, f_{2}=0 \text {, if either } t \leq 0 \text { or }|x|>R_{0},
$$

for some positive number $R_{0}$. We suppose that condition $(2.20)$ holds such that $a_{0}>0$. It can be checked that if $\tilde{x}$ is outward to the $2 \varepsilon_{0}$-vicinity of the point $O$, then $a_{i} \equiv 0, i=1,2$.

For the case $a_{i}=0, i=1,2$, problem (3.12)-(3.14) was studied by F. Yi [30] and the one-valued solvability of the problem was proved in the class $C_{T}^{2+\alpha}\left(R_{ \pm}^{2}\right)=$ $C\left([0, T] ; C^{2+\alpha}\left(R_{ \pm}^{2}\right)\right), \alpha \in(0,1)$. Here we will prove the one-to-one solvability of (3.12)-(3.14) in the class $E^{2+\alpha, \alpha, \alpha}$ if $a_{i} \neq 0, i=1,2$.

First of all, we study problem (3.12)-(3.14) in the special case

$$
f_{0}^{ \pm}=f_{2} \equiv 0
$$


and construct the integral representation to the solution $\left(u_{+}\left(x_{1}, x_{2}, t\right), u_{-}\left(x_{1}, x_{2}, t\right)\right)$. We denote by $\tilde{u}\left(\lambda, x_{2}, t\right)$ the Fourier transformation of the function $u\left(x_{1}, x_{2}, t\right)$, and by $\hat{u}(\cdot, \nu)$ the Laplace transformation of $u(\cdot, t)$, and use the notation " *" instead of " ". By applying the Fourier and Laplace transformations to problem (3.12)-(3.14), we get

$$
\begin{gathered}
\frac{\partial^{2} u_{ \pm}^{*}}{\partial x_{2}^{2}}-\lambda^{2} u_{ \pm}^{*}=0, \quad u_{ \pm}^{*}\left(\lambda, x_{2}, 0\right)=0 \\
\nu a_{0}\left[u_{-}^{*}\left(\lambda, x_{2}, \nu\right)-u_{+}^{*}\left(\lambda, x_{2}, \nu\right)\right]-\frac{\partial}{\partial x_{2}}\left[u_{-}^{*}\left(\lambda, x_{2}, \nu\right)-u_{+}^{*}\left(\lambda, x_{2}, \nu\right)\right] \\
+i a_{1} \lambda\left[u_{-}^{*}\left(\lambda, x_{2}, \nu\right)-u_{+}^{*}\left(\lambda, x_{2}, \nu\right)\right]=f_{1}^{*}(\lambda, \nu) \text { on } x_{2}=0 ; \\
\frac{\partial u_{-}^{*}\left(\lambda, x_{2}, \nu\right)}{\partial x_{2}}-k \frac{\partial u_{+}^{*}\left(\lambda, x_{2}, \nu\right)}{\partial x_{2}}+i k a_{2} \lambda\left[u_{-}^{*}\left(\lambda, x_{2}, \nu\right)-u_{+}^{*}\left(\lambda, x_{2}, \nu\right)\right]=0 \text { on } x_{2}=0 .
\end{gathered}
$$

To satisfy equations in (3.17), we set

$$
u_{-}^{*}\left(\lambda, x_{2}, \nu\right)=M_{-}(\lambda, \nu) e^{|\lambda| x_{2}}, \quad u_{+}^{*}\left(\lambda, x_{2}, \nu\right)=M_{+}(\lambda, \nu) e^{-|\lambda| x_{2}} ;
$$

and to find the unknown functions $M_{-}, M_{+}$, we have two transmission equations (3.18) and (3.19). It is easy to show that

$$
\begin{aligned}
M_{-}(\lambda, \nu)= & -k \frac{|\lambda|-i a_{2} \lambda}{|\lambda|+i k a_{2} \lambda} M_{+}(\lambda, \nu) \text {, and } M_{+}(\lambda, \nu)\left[1+k \frac{|\lambda|-i a_{2} \lambda}{|\lambda|+i k a_{2} \lambda}\right] \\
& \times\left[-\nu a_{0}-i a_{1} \lambda-|\lambda| \frac{1-k \frac{|\lambda|-i a_{2} \lambda}{|\lambda|+i k a_{2} \lambda}}{1+k \frac{|\lambda|-i a_{2} \lambda}{|\lambda|+i k a_{2} \lambda}}\right]=f_{1}^{*}(\lambda, \nu) .
\end{aligned}
$$

Thus, after some simple calculations in the last equation, one can get

$$
\begin{gathered}
M_{+}(\lambda, \nu)=\frac{1}{Q(\lambda, \nu)} \frac{1+i k a_{2} \frac{\lambda}{|\lambda|}}{k+1} f_{1}^{*}(\lambda, \nu), \\
M_{-}(\lambda, \nu)=-\frac{1}{Q(\lambda, \nu)} \frac{k\left(1-i a_{2} \frac{\lambda}{|\lambda|}\right)}{k+1} f_{1}^{*}(\lambda, \nu),
\end{gathered}
$$

where

$$
Q(\lambda, \nu)=-\nu a_{0}+\frac{k-1}{k+1}|\lambda|-i \lambda\left(a_{1}+\frac{2 k a_{2}}{1+k}\right) .
$$

Note that if condition(2.20) holds, then $-a_{0}<0$ and $\operatorname{Re} Q(\lambda, \nu)<0$ in the case of $\operatorname{Re} \nu>0$ and $\operatorname{Im} \lambda=0$. Hence, the function $\frac{1}{Q(\lambda, \nu)}$ does not have any singularities in this case. 
Let

$$
\begin{gathered}
F_{+}^{*}(\lambda, \nu)=-\frac{f_{1}^{*}(\lambda, \nu)}{a_{0}(k+1)}\left(1+i k a_{2} \frac{\lambda}{|\lambda|}\right), \quad F_{-}^{*}(\lambda, \nu)=\frac{k f_{1}^{*}(\lambda, \nu)}{a_{0}(k+1)}\left(1-i a_{2} \frac{\lambda}{|\lambda|}\right), \\
Q_{1}(\lambda, \nu)=\nu+A_{1}|\lambda|+i A_{2} \lambda,
\end{gathered}
$$

where $A_{1}=\frac{1-k}{a_{0}(k+1)}$ and $A_{2}=\frac{1}{a_{0}}\left(a_{1}+\frac{2 k a_{2}}{1+k}\right)$. Then the functions $M_{+}$and $M_{-}$ can be rewritten as

$$
M_{+}(\lambda, \nu)=\frac{F_{+}^{*}(\lambda, \nu)}{Q_{1}(\lambda, \nu)}, M_{-}(\lambda, \nu)=\frac{F_{-}^{*}(\lambda, \nu)}{Q_{1}(\lambda, \nu)}
$$

and the solution $\left(u_{+}^{*}, u_{-}^{*}\right)$ is

$$
u_{+}^{*}\left(\lambda, x_{2}, \nu\right)=\frac{F_{+}^{*}(\lambda, \nu)}{Q_{1}(\lambda, \nu)} e^{-|\lambda| x_{2}}, u_{-}^{*}\left(\lambda, x_{2}, \nu\right)=\frac{F_{-}^{*}(\lambda, \nu)}{Q_{1}(\lambda, \nu)} e^{|\lambda| x_{2}} .
$$

Thus, after the inverse Laplace and Fourier transformations, the solution $\left(u_{-}^{*}, u_{+}^{*}\right)$ gets the form

$$
u_{ \pm}\left(x_{1}, x_{2}, t\right)=\int_{0}^{t} d \tau \int_{-\infty}^{+\infty} F_{ \pm}\left(x_{1}-\xi, t-\tau\right) K_{ \pm}\left(\xi, x_{2}, \tau\right) d \xi
$$

where $K_{ \pm}\left(x_{1}, x_{2}, t\right)$ are the inverse Fourier and Laplace transformations of the function $\frac{e^{ \pm|\lambda| x_{2}}}{Q_{1}(\lambda, \nu)}$. Introduce the notation

$$
K_{+}\left(x_{1}, 0, t\right)=K_{-}\left(x_{1}, 0, t\right):=K\left(x_{1}, t\right) .
$$

As for the functions $F_{ \pm}\left(x_{1}, t\right)$, they are the inverse Fourier and Laplace transformations of $F_{ \pm}^{*}(\lambda, \nu)$. The results of Privalov's theorem for the singular integral (see, e.g., Theorem 15.3 in [11] or Theorem 3.1.1 in [10]) together with the properties of the function $f_{1}\left(x_{1}, t\right)$ give the following:

$$
\left\|F_{ \pm}\right\|_{E^{1+\alpha, \alpha, \alpha}\left(\overline{R_{T}^{1}}\right)} \leq \text { const }\left\|f_{1}\right\|_{E^{1+\alpha, \alpha, \alpha}\left(\overline{R_{T}^{1}}\right)} .
$$

To estimate the functions $u_{ \pm}\left(x_{1}, x_{2}, t\right)$, we need the following properties of the kernel $K\left(x_{1}, t\right)$.

Lemma 3.1. Let $\alpha \in(0,1), t \in[0, T], \Delta x=\bar{x}_{1}-x_{1}$ for every $\bar{x}_{1}, x_{1} \in R^{1}$, then the following holds:

i)

$$
K\left(x_{1}, t\right)=\frac{2}{A_{1} t\left(1+\left[\frac{x_{1}-A_{2} t}{A_{1} t}\right]^{2}\right)}, \quad K\left(x_{1}, t\right)>0
$$


ii)

$$
\int_{0}^{t} d \tau \int_{-\infty}^{+\infty} \frac{\partial^{l} K(y, \tau)}{\partial y^{l}} d y= \begin{cases}2 \pi t, & l=0 \\ 0, & l>0\end{cases}
$$

iii)

$$
I_{1}:=\int_{0}^{t} d \tau \int_{-\infty}^{+\infty}|y|^{\alpha}\left|\frac{\partial K(y, \tau)}{\partial y}\right| d y \leq \operatorname{const} t^{\alpha}
$$

$i v)$

$$
I_{2}:=\int_{0}^{t} d \tau \int_{\left|y-A_{2} \tau\right|<2|\Delta x|}|y|^{\alpha}\left|\frac{\partial K(y, \tau)}{\partial y}\right| d y \leq \text { const }|\Delta x|^{\alpha}
$$

$v)$

$$
I_{3}:=|\Delta x| \int_{0}^{t} d \tau \int_{\left|y-A_{2} \tau\right|>2|\Delta x|}|y|^{\alpha}\left|\frac{\partial^{2} K(y, \tau)}{\partial y^{2}}\right| d y \leq \text { const }|\Delta x|^{\alpha} .
$$

The proof of this lemma is technically tedious and we place it in Appendix.

$\mathrm{R}$ e $\mathrm{m}$ a $\mathrm{r} \mathrm{k}$ 3.1. By using the method of the proof of Lemma 3.1, it is not difficult to get analogous results for the function $K_{ \pm}\left(x_{1}, x_{2}, t\right)$.

Thus the standard arguments of Chapter 4 [22], together with Lemma 3.1, Remark 3.1 and estimate (3.27), after routine calculations lead to the inequalities

$$
\begin{gathered}
\left\|u_{ \pm}\right\|_{E^{2+\alpha, \alpha, \alpha}\left(\overline{R_{ \pm T}^{2}}\right)}+\left\|\frac{\partial u_{ \pm}}{\partial t}\right\|_{E^{1+\alpha, \alpha, \alpha}\left(\overline{R_{T}^{1}}\right)} \leq \mathrm{const}\left\|f_{1}\right\|_{E^{1+\alpha, \alpha, \alpha}\left(\overline{R_{T}^{1}}\right)}, \\
\left\|u_{ \pm}\right\|_{E^{1+\alpha, \alpha, \alpha}\left(\overline{R_{+T}^{2}}\right)} \leq \mathrm{const} T^{\alpha^{*}-\alpha}\left\|f_{1}\right\|_{E^{1+\alpha, \alpha, \alpha}\left(\overline{R_{T}^{1}}\right)},
\end{gathered}
$$

where $0<\alpha<\alpha^{*} \leq 1$. Note that in the case of $f_{0}^{ \pm}=f_{2}=0$, the uniqueness of the solution $\left(u_{-}(x, t), u_{+}(x, t)\right)$ to problem (3.12)-(3.14) follows immediately from the first inequality in (3.33). Therefore, in the case of (3.16), the one-valued solvability of problem (3.12)-(3.13) was proved and the corresponding coercive estimates were obtained. To extend these results to the general case, i.e., $f_{0}^{ \pm} \neq 0$, $f_{2} \neq 0$, we look for the solution of problem (3.12)-(3.13) in the form

$$
u_{ \pm}(x, t)=\bar{u}_{ \pm}(x, t)+\overline{\bar{u}}_{ \pm}(x, t)
$$


where $\bar{u}_{ \pm}(x, t)$ is the solution of the transmission problem

$$
\begin{gathered}
\Delta \bar{u}_{ \pm}=f_{0}^{ \pm} \text {in } R_{ \pm T}^{2}, \quad \bar{u}_{ \pm}(x, 0)=0, x \in R_{ \pm}^{2} ; \\
\bar{u}_{+}=\bar{u}_{-}, \frac{\partial \bar{u}_{-}}{\partial n}-k \frac{\partial \bar{u}_{+}}{\partial n}=f_{2}, \quad(x, t) \in \overline{R_{T}^{1}}
\end{gathered}
$$

and $\overline{\bar{u}}_{ \pm}(x, t)$ is the solution of problem (3.12)-(3.13) in the case (3.16) with a new right-hand side $f_{1}$.

As for $\bar{u}_{ \pm}(x, t)$, we apply the well-known results from [27] which give

$$
\left\|\bar{u}_{ \pm}\right\|_{C^{2+\alpha}\left(\overline{R_{ \pm T}^{2}}\right)} \leq \mathrm{const}\left[\left\|f_{0}^{ \pm}\right\|_{C^{\alpha}\left(\overline{R_{ \pm T}^{2}}\right)}+\left\|f_{2}\right\|_{C^{1+\alpha}\left(\overline{R_{T}^{1}}\right)}\right] .
$$

The corresponding smoothness of the functions $\bar{u}_{ \pm}(x, t)$ with respect to $t$ is obtained if one considers problem (3.34) for the functions $U_{ \pm}=\bar{u}_{ \pm}\left(x, t_{1}\right)-\bar{u}_{ \pm}\left(x, t_{2}\right)$ with new right-hand sides: $\bar{f}_{0}^{ \pm}=f_{0}^{ \pm}\left(x, t_{1}\right)-f_{0}^{ \pm}\left(x, t_{2}\right) ; \bar{f}_{2}=f_{2}\left(x, t_{1}\right)-f_{2}\left(x, t_{2}\right)$. Inequality (3.35) in the case of functions $U_{ \pm}$together with the properties of the functions $f_{0}^{ \pm}, f_{2}$ lead to the estimate

$$
\left\|\bar{u}_{ \pm}\right\|_{E^{2+\alpha, \alpha, \alpha}\left(\overline{R_{ \pm T}^{2}}\right)} \leq \text { const }\left[\left\|f_{0}^{ \pm}\right\|_{C^{\alpha, \alpha, \alpha}\left(\overline{R_{ \pm T}^{2}}\right)}+\left\|f_{2}\right\|_{C^{1+\alpha, \alpha, \alpha}\left(\overline{R_{T}^{1}}\right)}\right] .
$$

Moreover, as it follows from the third condition in (3.34),

$$
\frac{\partial \bar{u}_{-}}{\partial t}-\frac{\partial \bar{u}_{+}}{\partial t}=0 \text { if }(x, t) \in \overline{R_{T}^{1}}
$$

Thus, the all written above proves the following results.

Theorem 3.3. Let $\alpha \in(0,1), f_{0}^{ \pm} \in E^{\alpha, \alpha, \alpha}\left(R_{ \pm T}^{2}\right), f_{i} \in E^{1+\alpha, \alpha, \alpha}\left(\overline{R_{T}^{1}}\right), i=$ 1,2 , and condition (3.15) hold. Then there exists a unique solution $u_{ \pm}(x, t) \in$ $E^{2+\alpha, \alpha, \alpha}\left(\overline{\left.R_{ \pm T}^{2}\right)}\right.$ of problem (3.12)-(3.14), and the estimate is true

$$
\begin{gathered}
\left\|u_{+}\right\|_{E^{2+\alpha, \alpha, \alpha}\left(\overline{R_{+T}^{2}}\right)}+\left\|u_{-}\right\|_{E^{2+\alpha, \alpha, \alpha}\left(\overline{R_{-T}^{2}}\right)}+\left\|\frac{\partial u_{-}}{\partial t}-\frac{\partial u_{+}}{\partial t}\right\|_{E^{1+\alpha, \alpha, \alpha}\left(\overline{R_{T}^{1}}\right)} \\
\leq c\left[\left\|f_{0}^{+}\right\|_{C^{\alpha, \alpha, \alpha}\left(\overline{R_{+T}^{2}}\right)}+\left\|f_{0}^{-}\right\|_{C^{\alpha, \alpha, \alpha}\left(\overline{R_{-T}^{2}}\right)}+\left\|f_{1}\right\|_{E^{1+\alpha, \alpha, \alpha}\left(\overline{R_{T}^{1}}\right)}+\left\|f_{2}\right\|_{C^{1+\alpha, \alpha, \alpha}\left(\overline{R_{T}^{1}}\right)}\right],
\end{gathered}
$$

where $c$ is a positive constant which is independent of the right-hand sides in (3.12)-(3.14).

Moreover, if in addition condition (3.16) holds, then inequities (3.33) take place. 


\section{The Linear Problem}

As it follows from (2.33)-(2.38), the linear system corresponding to nonlinear system (2.39), where the right-hand sides are some fixed functions, has the form

$$
\begin{gathered}
\frac{\partial^{2} \theta_{i}}{\partial x_{1}^{2}}+\frac{\partial^{2} \theta_{i}}{\partial x_{2}^{2}}=f_{0 i}(x, t) \quad \text { in } \Omega_{i T}, \\
\theta_{1}(x, t)-\theta_{2}(x, t)=A(x) \sigma \quad \text { on } \Gamma_{T}, \\
\frac{\partial \sigma}{\partial t}=-k_{1} a(x) \frac{\partial \theta_{1}}{\partial n}-k_{1} A_{1}(x)\left(\frac{\partial \theta_{1}}{\partial \omega}-\frac{\partial \theta_{2}}{\partial \omega}\right)+f_{1}(x, t) \quad \text { on } \Gamma_{T}, \\
\frac{\partial \theta_{1}}{\partial n}-k \frac{\partial \theta_{2}}{\partial n}+A_{2}(x)\left(\frac{\partial \theta_{1}}{\partial \omega}-\frac{\partial \theta_{2}}{\partial \omega}\right)=f_{2}(x, t) \quad \text { on } \Gamma_{T}, \\
\left.\frac{\partial \theta_{i}}{\partial x_{1}}\right|_{x_{1}=0}=0, \theta_{i}(x, t)=0 \quad \text { on } \quad \Gamma_{i T}, \\
\sigma(\omega, 0)=0, \quad \theta_{i}(x, 0)=0 .
\end{gathered}
$$

Here $f_{0 i}(x, t), f_{i}(x, t), a(x), A(x), A_{1}(x), A_{2}(x)$ are some given functions $A(x)<0$, and

$$
f_{0 i}(x, 0)=0, x \in \Omega_{i}, i=1,2, f_{j}(x, 0)=0, x \in \Gamma, j=1,2 .
$$

In the $2 \varepsilon_{0}$-vicinity of the corner point $O$, the coefficients $a(x), A(x), A_{1}(x), A_{2}(x)$ can be represented as (see (2.65), (2.66) and (2.68))

$$
\begin{gathered}
A(x)=\frac{1-k}{k} \frac{1}{\sqrt{1+\varphi_{x_{1}}^{2}}} \frac{\partial v_{10}}{\partial n}, \quad A_{1}(x)=\frac{k_{2}}{k-1} \sqrt{1+\varphi_{x_{1}}^{2}}\left[\frac{\left.\frac{\partial v_{10}}{\frac{\partial \omega}{\partial v_{10}}}+\varphi_{x_{1}}\right],}{a(x)=\sqrt{1+\varphi_{x_{1}}^{2}}, \quad A_{2}(x)=-k \frac{\frac{\partial v_{10}}{\frac{\partial \omega}{\partial v_{10}}}}{\partial n}}\right.
\end{gathered}
$$

where (see (2.18)) if $x \rightarrow O$

$$
\left.\frac{\partial v_{10}}{\partial n}\right|_{\Gamma_{T}} \sim A_{3} r^{1+\gamma},\left.\frac{\partial v_{10}}{\partial \omega}\right|_{\Gamma_{T}} \sim A_{4} r^{1+\gamma},
$$

where $\gamma$ is given in (2.19), $A_{3}$ and $A_{4}$ are nonzero constants, $A_{3}<0$. As it follows from (2.18),

$$
a(x), A_{i}(x) \in C^{2+\alpha}(\bar{\Gamma}), i=1,2 \text {, and } A(x) \in E_{1+\gamma}^{2+\alpha}(\bar{\Gamma}) .
$$

We introduce the functional spaces $H_{D}$ and $H_{R}$ (such that $\mathbf{z} \in H_{D}, \overline{\mathrm{F}}(\mathbf{z}) \in H_{R}$ ),

$$
H_{D}=E_{s}^{2+\alpha, \alpha, \alpha}\left(\bar{\Omega}_{1 T}\right) \times E_{s}^{2+\alpha, \alpha, \alpha}\left(\bar{\Omega}_{2 T}\right) \times N_{s, \gamma}^{2+\alpha}\left(\bar{\Gamma}_{T}\right),
$$




$$
H_{R}=E_{s-2}^{\alpha, \alpha, \alpha}\left(\bar{\Omega}_{1 T}\right) \times E_{s-2}^{\alpha, \alpha, \alpha}\left(\bar{\Omega}_{2 T}\right) \times E_{s-1}^{1+\alpha, \alpha, \alpha}\left(\bar{\Gamma}_{T}\right) \times E_{s-1}^{1+\alpha, \alpha, \alpha}\left(\bar{\Gamma}_{T}\right),
$$

and

$$
\begin{gathered}
\|\mathbf{z}\|_{H_{D}}=\left\|\left(\theta_{1}, \theta_{2}, \sigma\right)\right\|_{H_{D}}=\left\|\theta_{1}\right\|_{E_{s}^{2+\alpha, \alpha, \alpha}\left(\bar{\Omega}_{1 T}\right)}+\left\|\theta_{2}\right\|_{E_{s}^{2+\alpha, \alpha, \alpha}\left(\bar{\Omega}_{2 T}\right)}+\|\sigma\|_{N_{s, \gamma}^{2+\alpha}\left(\bar{\Gamma}_{T}\right)} \\
\|\overline{\mathrm{F}}(\mathbf{z})\|_{H_{R}}=\left\|\left(f_{01}, f_{02}, f_{1}, f_{2}\right)\right\|_{H_{R}}=\left\|f_{01}\right\|_{E_{s-2}^{\alpha, \alpha, \alpha}\left(\bar{\Omega}_{1 T}\right)}+\left\|f_{02}\right\|_{E_{s-2}^{\alpha, \alpha, \alpha}\left(\bar{\Omega}_{2 T}\right)} \\
+\left\|f_{1}\right\|_{E_{s-1}^{1+\alpha, \alpha, \alpha}\left(\bar{\Gamma}_{T}\right)}+\left\|f_{2}\right\|_{E_{s-1}^{1+\alpha, \alpha, \alpha}\left(\bar{\Gamma}_{T}\right)} .
\end{gathered}
$$

Theorem 4.1. Let $\left(f_{01}, f_{02}, f_{1}, f_{2}\right) \in H_{R}$ with $\alpha \in(0,1 / 2)$ and s satisfy conditions $i)$, ii) from Theorem 2.1, conditions (2.20) and (4.7)-(4.10) hold. Then for some $T$ there exists a unique solution $\left(\theta_{1}, \theta_{2}, \sigma\right) \in H_{D}$ to problem (4.1)(4.6) for $t \in[0, T]$, and

$$
\left\|\left(\theta_{1}, \theta_{2}, \sigma\right)\right\|_{H_{D}} \leq \mathrm{const}\left\|\left(f_{01}, f_{02}, f_{1}, f_{2}\right)\right\|_{H_{R}}
$$

with the constant independent of the functions $f_{01}, f_{02}, f_{1}, f_{2}$.

For the sake of simplicity, we represent the proof of Theorem 4.1 in the case of $\Gamma=\Gamma(0)$ described by the equation $x_{2}=x_{1} \tan \beta$ in the $2 \varepsilon_{0}$-vicinity of the corner point $O$. This theorem in general case $\left(x_{2}=\varphi\left(x_{1}\right)\right)$ is proved in the same way and with the arguments and transformations taken from [5] and [29].

Using the results from [8], we can reduce problem (4.1)-(4.6) to the similar one with $f_{01}=f_{02}=f_{2}=0$ and a new function $f_{1}(x, t) \in E_{s-1}^{1+\alpha, \alpha, \alpha}\left(\bar{\Gamma}_{T}\right)$. Indeed, let

$$
\theta_{i}(x, t)=\bar{\theta}_{i}(x, t)+\overline{\bar{\theta}}_{i}(x, t),
$$

where $\left(\overline{\bar{\theta}}_{1}(x, t), \overline{\bar{\theta}}_{2}(x, t)\right)$ is the solution of the problem

$$
\begin{gathered}
\frac{\partial^{2} \overline{\bar{\theta}}_{i}}{\partial x_{1}^{2}}+\frac{\partial^{2} \overline{\bar{\theta}}_{i}}{\partial x_{2}^{2}}=f_{0 i}(x, t) \quad \text { in } \Omega_{i T}, \\
\overline{\bar{\theta}}_{1}(x, t)-\overline{\bar{\theta}}_{2}(x, t)=0, \quad \frac{\partial \overline{\bar{\theta}}_{1}}{\partial n}-k \frac{\partial \overline{\bar{\theta}}_{2}}{\partial n}=f_{2}(x, t) \quad \text { on } \Gamma_{T}, \\
\left.\frac{\partial \overline{\bar{\theta}}_{i}}{\partial x_{1}}\right|_{x_{1}=0}=0, \quad \overline{\bar{\theta}}_{i}(x, t)=0 \text { on } \Gamma_{i T}, \overline{\bar{\theta}}_{i}(x, 0)=0 .
\end{gathered}
$$

Transmission problem (4.13), as follows from Theorem 1.1 and Remark 3.1 [8], has a unique solution $\overline{\bar{\theta}}_{i}(x, t) \in E_{s}^{2+\alpha}\left(\bar{\Omega}_{i T}\right), i=1,2$, and

$$
\begin{gathered}
\left\|\overline{\bar{\theta}}_{1}\right\|_{E_{s}^{2+\alpha}\left(\bar{\Omega}_{1 T}\right)}+\left\|\overline{\bar{\theta}}_{2}\right\|_{E_{s}^{2+\alpha}\left(\bar{\Omega}_{2 T}\right)} \leq \mathrm{const}\left(\left\|f_{01}\right\|_{E_{s-2}^{\alpha}\left(\bar{\Omega}_{1 T}\right)}\right. \\
\left.+\left\|f_{02}\right\|_{E_{s-2}^{\alpha}\left(\bar{\Omega}_{2 T}\right)}+\left\|f_{2}\right\|_{E_{s-1}^{1+\alpha}\left(\bar{\Gamma}_{T}\right)}\right)
\end{gathered}
$$


where $s$ satisfies the conditions from Theorem 4.1.

As for the estimates of $\overline{\bar{\theta}}_{i}, i=1,2$, with respect to $t$, they are a simple consequence of (4.14) if we consider problem (4.13) for the functions $\left[\overline{\bar{\theta}}_{i}\left(x, t_{1}\right)-\right.$ $\left.\overline{\bar{\theta}}_{i}\left(x, t_{2}\right)\right], i=1,2$, and use the properties of the right-hand side. Thus we have

$$
\begin{gathered}
\left\|\overline{\bar{\theta}}_{1}\right\|_{E_{s}^{2+\alpha, \alpha, \alpha}\left(\bar{\Omega}_{1 T}\right)}+\left\|\overline{\bar{\theta}}_{2}\right\|_{E_{s}^{2+\alpha, \alpha, \alpha}\left(\bar{\Omega}_{2 T}\right)} \leq \operatorname{const}\left(\left\|f_{01}\right\|_{E_{s-2}^{\alpha, \alpha, \alpha}\left(\bar{\Omega}_{1 T}\right)}\right. \\
\left.+\left\|f_{02}\right\|_{E_{s-2}^{\alpha, \alpha, \alpha}\left(\bar{\Omega}_{2 T}\right)}+\left\|f_{2}\right\|_{E_{s-1}^{1+\alpha, \alpha, \alpha}\left(\bar{\Gamma}_{T}\right)}\right) .
\end{gathered}
$$

For the functions $\bar{\theta}_{i}(x, t), i=1,2$, we get

$$
\begin{gathered}
\frac{\partial^{2} \bar{\theta}_{i}}{\partial x_{1}^{2}}+\frac{\partial^{2} \bar{\theta}_{i}}{\partial x_{2}^{2}}=0 \quad \text { in } \Omega_{i T}, \\
\bar{\theta}_{1}(x, t)-\bar{\theta}_{2}(x, t)-A(x) \sigma=0 \quad \text { on } \Gamma_{T}, \\
\frac{\partial \sigma}{\partial t}+k_{1} a(x) \frac{\partial \bar{\theta}_{1}}{\partial n}+k_{1} A_{1}(x)\left(\frac{\partial \bar{\theta}_{1}}{\partial \omega}-\frac{\partial \bar{\theta}_{2}}{\partial \omega}\right) \\
=f_{1}(x, t)-k_{1} a(x) \frac{\partial \bar{\theta}_{1}}{\partial n} \equiv \bar{f}_{1}(x, t) \quad \text { on } \Gamma_{T}, \\
\frac{\partial \bar{\theta}_{1}}{\partial n}-k \frac{\partial \bar{\theta}_{2}}{\partial n}+A_{2}(x)\left(\frac{\partial \bar{\theta}_{1}}{\partial \omega}-\frac{\partial \bar{\theta}_{2}}{\partial \omega}\right)=0 \quad \text { on } \Gamma_{T}, \\
\left.\frac{\partial \bar{\theta}_{i}}{\partial x_{1}}\right|_{x_{1}=0}=0, \bar{\theta}_{i}(x, t)=0 \quad \text { on } \quad \Gamma_{i T}, \\
\sigma(\omega, 0)=0, \quad \bar{\theta}_{i}(x, 0)=0 .
\end{gathered}
$$

Note that due to (4.15) and the properties of the function $f_{1}(x, t)$, the function $\bar{f}_{1}(x, t)$ belongs to $E_{s-1}^{1+\alpha, \alpha, \alpha}\left(\bar{\Gamma}_{T}\right)$ and $\bar{f}_{1}(x, 0)=0$.

Therefore it is enough to prove Theorem 4.1 for problem (4.16)-(4.21). This proof consists of two parts. The first of them is a solvability of problem (4.16)(4.21) which is done with the technique of the regularizer for parabolic systems taken from [22]. As for the uniqueness of the solutions (the second part of the proof of this theorem), it is deduced by the corresponding a priori estimates.

To show the solvability of system (4.16)-(4.21), we reduce one, similarly to [24] or [21], to the nonlocal equation

$$
\mathcal{L} \sigma=\bar{f}_{1} \text { on } \Gamma_{T},\left.\sigma\right|_{t=0}=0 \text { on } \Gamma \text {. }
$$

The operator $\mathcal{L}$ is constructed in the next way. Let $\sigma$ be given in equation (4.17). Then equations (4.16), (4.17), (4.19)-(4.21) formulate the transmission problem for the functions $\theta_{1}$ and $\theta_{2}$. The solution of this transmission problem can be used in equation (4.18). Thus, $\mathcal{L} \sigma$ is given by the left-hand side of (4.18). 
A nonlocal character of system (4.22) causes the difficulties requiring technical tricks related to a suitable localization. As well as in Chapter 4 from [22] or Section 2 from [24], or Section 4 from [21], we introduce the two collections of open sets: $\left\{\omega_{i}^{m}\right\}$ and $\left\{\Omega_{i}^{m}\right\}, i=1,2$, such that

$$
\begin{gathered}
\bar{\omega}_{i}^{m} \subset \Omega_{i}^{m} \subset \bar{\Omega}_{i}, \cup_{m} \omega_{i}^{m}=\cup_{m} \Omega_{i}^{m}=\bar{\Omega}_{i}, \\
\omega_{i}^{m}=B_{\lambda / 2}\left(x^{m}\right) \cap \bar{\Omega}_{i} ; \quad \Omega_{i}^{m}=B_{\lambda}\left(x^{m}\right) \cap \bar{\Omega}_{i}
\end{gathered}
$$

with $m=1, \ldots, N_{0}$ and $B_{\lambda}\left(x^{m}\right), B_{\lambda / 2}\left(x^{m}\right)$ being the balls with the center in $x^{m}$ and the radiuses of $\lambda$ and $\lambda / 2$, respectively. Denote $\Gamma^{m}=\Gamma \cap B_{\lambda}\left(x^{m}\right)$ and $\Gamma_{i}^{m}=\Gamma_{i} \cap B_{\lambda}\left(x^{m}\right), i=1,2$. The index $m$ belongs to one of two sets:

$$
m \in M \text { if } \bar{\Omega}_{i}^{m} \cap \Gamma=\emptyset \text { and } m \in N \text { if } \bar{\omega}_{i}^{m} \cap \Gamma \neq \emptyset .
$$

We say that $m \in N_{1}$ if $m$ belongs to $N$ and $\Gamma^{m} \cap B_{\varepsilon_{0}}(0) \neq \emptyset\left(B_{\varepsilon_{0}}(0)\right.$ is the ball with the center in $O$ and the $\varepsilon_{0}$ radius), and $N_{2}=N \backslash N_{1}$. Moreover, $N_{2}=N_{21} \cup N_{22}$, where $m \in N_{21}$ if $m \in N_{2}$ and $\Gamma^{m} \cap B_{2 \varepsilon_{0}}(0) \neq \emptyset\left(B_{2 \varepsilon_{0}}(0)\right.$ is the ball with the center in $O$ and the $2 \varepsilon_{0}$ radius).

The coverings $\left\{\omega_{i}^{m}\right\}$ and $\left\{\Omega_{i}^{m}\right\}, i=1,2$, define a partition of unity for the domains $\Omega_{i}$. Let $\xi_{i}^{m}: \Omega_{i} \rightarrow[0,1], i=1,2$, be a smooth function such that

$$
\xi_{i}^{m}=1 \text {, if } x \in \omega_{i}^{m}, \xi_{i}^{m}=0 \text {, if } x \in \Omega_{i} \backslash \Omega_{i}^{m}, \xi_{i}^{m} \in(0,1) \text {, if } x \in \Omega_{i}^{m} \backslash \omega_{i}^{m},
$$

and $\left|\nabla^{|l|} \xi_{i}^{m}\right| \leq$ const $\lambda^{-|l|}, 1 \leq \sum_{m}\left(\xi_{i}^{m}\right)^{2} \leq M_{0}$. By using the functions $\xi_{i}^{m}$, we define the function

$$
\eta_{i}^{m}=\frac{\xi_{i}^{m}}{\sum_{j}\left(\xi_{i}^{j}\right)^{2}} .
$$

By the properties of the functions $\xi_{i}^{m}$, the functions $\eta_{i}^{m}$ vanish for $x \in \Omega_{i} \backslash \Omega_{i}^{m}$; in addition, $\left|\nabla^{|l|} \eta_{i}^{m}\right| \leq$ const $\lambda^{-|l|}$.

The functions $\eta_{i}^{m} \xi_{i}^{m}$ define the partition of unity by the formula

$$
\sum_{m} \eta_{i}^{m} \xi_{i}^{m}=1, \quad i=1,2 .
$$

Note that if $m \in N$, we can choose the same functions $\xi^{m}$ for both $i=1$ and $i=2$, and thus $\eta_{1}^{m}=\eta_{2}^{m} \equiv \eta^{m}, m \in N$.

For each $m \in N$, we pick out one point $x^{m} \in \omega_{i}^{m} \cap \Gamma$ which will be the origin of the local coordinate system. The description of this system can be found in Chapter 4 [22], Section 2 [24].

We introduce local coordinate systems connected with each point $x^{m}, m \in N_{2}$. Let the curve $\Gamma$ be described with $y_{2}=\Psi^{m}\left(y_{1}\right)$ in a small vicinity of every point $x^{m}, m \in N_{2}$, and

$$
y=B^{(m)}\left(x-x^{m}\right), \quad\left|\Psi^{m}\left(y_{1}\right)\right| \leq \text { const } \lambda,
$$


where $B^{(m)}=\left(B_{i j}^{(m)}\right)_{i, j=1,2}$ is an orthogonal matrix. After that the local "flatness" of the boundary is made with the change of variables

$$
z_{1}=y_{1}, z_{2}=y_{2}-\Psi^{m}\left(y_{1}\right), m \in N_{22} ; z_{1}=y_{1}, z_{2}=y_{2}, m \in N_{21} .
$$

Thus, the variables $\left(x_{1}, x_{2}\right)$ are connected with $\left(z_{1}, z_{2}\right)$ by the maps $Z^{m}$ (see (4.23), (4.24)) such that

$$
x=Z_{m}(z) \text {, and } z=Z_{m}^{-1}(x), m \in N_{2} .
$$

Definition 4.1. An operator

$$
\mathcal{R}: E_{s-1}^{1+\alpha, \alpha, \alpha}\left(\bar{\Gamma}_{T}\right) \rightarrow N_{s, \gamma}^{2+\alpha}\left(\bar{\Gamma}_{T}\right)
$$

such that

$$
\mathcal{R} \bar{f}_{1}=\sum_{m \in N} \eta^{m} s^{m}
$$

is called a regularizer where $s^{m}$ is a solution of the following problems: if $m \in N_{1}$, then

$$
\begin{gathered}
\frac{\partial^{2} \bar{w}_{i}^{m}}{\partial x_{1}^{2}}+\frac{\partial^{2} \bar{w}_{i}^{m}}{\partial x_{2}^{2}}=0 \quad \text { in } G_{i T}, \\
\bar{w}_{1}^{m}(x, t)-\bar{w}_{2}^{m}(x, t)-A^{m} s^{m}=0 \quad \text { on } g_{T}, \\
\frac{\partial s^{m}}{\partial t}+k_{1} a^{m} \frac{\partial \bar{w}_{1}^{m}}{\partial n}+k_{1} A_{1}^{m}\left(\frac{\partial \bar{w}_{1}^{m}}{\partial \omega}-\frac{\partial \bar{w}_{2}^{m}}{\partial \omega}\right)=\bar{f}_{1}^{m}(x, t) \quad \text { on } g_{T}, \\
\frac{\partial \bar{w}_{1}^{m}}{\partial n}-k \frac{\partial \bar{w}_{2}^{m}}{\partial n}+A_{2}^{m}\left(\frac{\partial \bar{w}_{1}^{m}}{\partial \omega}-\frac{\partial \bar{w}_{2}^{m}}{\partial \omega}\right)=0 \quad \text { on } g_{T}, \\
\frac{\partial \bar{w}_{1}^{m}}{\partial n}=0 \text { on }\left\{x_{1}=0, x_{2}<0\right\} \times[0, T] ; \quad \frac{\partial \bar{w}_{2}^{m}}{\partial n}=0 \text { on }\left\{x_{1}=0, x_{2}>0\right\} \times[0, T], \\
s^{m}(\omega, 0)=0, \quad \bar{w}_{i}^{m}(x, 0)=0,
\end{gathered}
$$

where $G_{i T}$ and $g_{T}$ are defined in Subsection 3.1;

if $m \in N_{2}$, then $s^{m}(\omega(x), t):=\hat{s}^{m}\left(\hat{\omega}\left(Z_{m}^{-1}(x)\right), t\right)$ and

$$
\begin{gathered}
\frac{\partial^{2} \hat{w}_{i}^{m}}{\partial z_{1}^{2}}+\frac{\partial^{2} \hat{w}_{i}^{m}}{\partial z_{2}^{2}}=0 \quad \text { in } R_{ \pm T}^{2}, \\
\hat{w}_{1}^{m}(z, t)-\hat{w}_{2}^{m}(z, t)-A^{m} \hat{s}^{m}=0 \quad \text { on } R_{T}^{1}, \\
\frac{\partial \hat{s}^{m}}{\partial t}+k_{1} a^{m} \frac{\partial \hat{w}_{1}^{m}}{\partial \hat{n}}+k_{1} A_{1}^{m}\left(\frac{\partial \hat{w}_{1}^{m}}{\partial \hat{\omega}}-\frac{\partial \hat{w}_{2}^{m}}{\partial \hat{\omega}}\right)=\hat{f}_{1}^{m}(z, t) \quad \text { on } R_{T}^{1},
\end{gathered}
$$




$$
\begin{gathered}
\frac{\partial \hat{w}_{1}^{m}}{\partial \hat{n}}-k \frac{\partial \hat{w}_{2}^{m}}{\partial \hat{n}}+A_{2}^{m}\left(\frac{\partial \hat{w}_{1}^{m}}{\partial \hat{\omega}}-\frac{\partial \hat{w}_{2}^{m}}{\partial \hat{\omega}}\right)=0 \quad \text { on } R_{T}^{1}, \\
\hat{s}^{m}(\omega, 0)=0, \quad \hat{w}_{i}^{m}(z, 0)=0,
\end{gathered}
$$

where $\hat{\omega}:=\hat{\omega}(z)$ and $\hat{n}:=\hat{n}(z)$ are the unit vectors with the coordinates $\{1,0\}$ and $\{0,-1\}$, respectively, in the plane $R^{2}$, i.e., $\frac{\partial}{\partial \hat{n}}=-\frac{\partial}{\partial z_{2}}$ and $\frac{\partial}{\partial \hat{\omega}}=\frac{\partial}{\partial z_{1}}$. Here

$$
\begin{gathered}
\bar{f}_{1}^{m}=\bar{f}_{1} \xi^{m}, \hat{f}_{1}^{m}(z, t)=\bar{f}_{1}^{m}\left(Z_{m}(z), t\right), a^{m}=a\left(x^{m}\right), A_{i}^{m}=A_{i}\left(x^{m}\right), i=1,2 \\
A^{m}=A\left(x^{m}\right) \leq 0, \text { if } m \in N_{2}, \text { and } \text { for } m \in N_{1} A^{m}=r^{1+\gamma} \tilde{A}^{m} \\
\tilde{A}^{m}=\frac{1-k}{k} \frac{1}{\sqrt{1+\varphi_{x_{1}}^{2}\left(x^{m}\right)}} A_{3}
\end{gathered}
$$

where $A_{3}$ is a negative constant from (4.9).

Note that after simple transformations similar to those from Subsection 2.4 (see (2.65)-(2.78)), the results of Section 3 are applied to the solutions of problems (4.26) and (4.27).

The operator $\mathcal{R}$ enables to construct an inverse operator to $\mathcal{L}$ by the methods used in Section 4 [21]. First, we need the following result.

Lemma 4.1. Let the conditions of Theorem 4.1 hold, and $\overline{\boldsymbol{F}}(\mathbf{z})=\left(0,0, \bar{f}_{1}, 0\right)$, $\bar{f}_{1}(x, t) \in E_{s-1}^{1+\alpha, \alpha, \alpha}\left(\bar{\Gamma}_{T}\right), \bar{f}_{1}(x, 0)=0$. Then

$$
\mathcal{L} \mathcal{R} \bar{f}_{1}=\bar{f}_{1}+\mathcal{T} \bar{f}_{1}
$$

and the norm of the operator $\mathcal{T}$ is small and controlled by a quantity $C(\lambda, T)$. If the time interval and $\lambda$ tend to zero, then $C(\lambda, T)$ vanishes.

$\mathrm{P}$ r o o f. We will use below the notation $\bar{w}_{i}^{m}(x, t):=\hat{w}_{i}^{m}\left(Z_{m}^{-1}(x), t\right)$, $n:=\hat{n}\left(Z_{m}^{-1}(x)\right), \omega:=\hat{\omega}\left(Z_{m}^{-1}(x)\right) m \in N_{2}$.

Let us introduce the auxiliary functions

$$
v_{\bar{f}_{1}}^{i}=\sum_{m \in N} \eta^{m} \bar{w}_{i}^{m}, i=1,2,
$$

which are the solution of the transmission problem

$$
\begin{gathered}
\Delta_{x} v_{\bar{f}_{1}}^{i}=\sum_{m \in N}\left[2 \nabla_{x} \eta^{m} \nabla_{x} \bar{w}_{i}^{m}+\bar{w}_{i}^{m} \Delta_{x} \eta^{m}\right]+\sum_{m \in N_{22}} \eta^{m} \Delta_{x} \bar{w}_{i}^{m}(x) \equiv g_{0 i} i n \Omega_{i T}, \\
v_{\bar{f}_{1}}^{1}-v_{\bar{f}_{1}}^{2}=\sum_{m \in N} A^{m} s^{m} \eta^{m}=\sum_{m \in N}\left[A^{m}-A(x)\right] s^{m} \eta^{m}+A(x) \sum_{m \in N} s^{m} \eta^{m}
\end{gathered}
$$




$$
\begin{gathered}
=\sum_{m \in N}\left[A^{m}-A(x)\right] s^{m} \eta^{m}+A(x) \mathcal{R} \bar{f}_{1} \equiv g_{1} \text { on } \Gamma_{T} \\
\frac{\partial v_{\bar{f}_{1}}^{1}}{\partial n}-k \frac{\partial v_{\bar{f}_{1}}^{2}}{\partial n}=-\sum_{m \in N} A_{2}^{m}\left[\frac{\partial \bar{w}_{1}^{m}}{\partial \omega}-\frac{\partial \bar{w}_{2}^{m}}{\partial \omega}\right] \eta^{m}+\sum_{m \in N}\left[\bar{w}_{1}^{m}-k \bar{w}_{2}^{m}\right] \frac{\partial \eta^{m}}{\partial n} \\
=-A_{2}(x) \sum_{m \in N}\left[\frac{\partial \bar{w}_{1}^{m}}{\partial \omega}-\frac{\partial \bar{w}_{2}^{m}}{\partial \omega}\right] \eta^{m}+\sum_{m \in N}\left[A_{2}(x)-A_{2}^{m}\right]\left[\frac{\partial \bar{w}_{1}^{m}}{\partial \omega}-\frac{\partial \bar{w}_{2}^{m}}{\partial \omega}\right] \eta^{m} \\
+\sum_{m \in N}\left[\bar{w}_{1}^{m}-k \bar{w}_{2}^{m}\right] \frac{\partial \eta^{m}}{\partial n} \equiv g_{2} \quad \text { on } \quad \Gamma_{T}, \\
v_{\bar{f}_{1}}^{i}=0 \quad \text { on } \quad \Gamma_{i T}, \quad v_{\bar{f}_{1}}^{i}(x, 0)=0 .
\end{gathered}
$$

As for solvability of problem (4.31)-(4.34), we can apply the results of Theorem 1.1 and Remark 3.1 from [8] to obtain the existence of the unique solution $\left(v_{\bar{f}_{1}}^{1}, v_{\bar{f}_{1}}^{2}\right)$,

$$
\sum_{i=1}^{2}\left\|v_{\bar{f}_{1}}^{i}\right\|_{E_{s}^{2+\alpha}\left(\bar{\Omega}_{i T}\right)} \leq \operatorname{const}\left[\sum_{i=1}^{2}\left\|g_{0 i}\right\|_{E_{s-2}^{\alpha}\left(\bar{\Omega}_{i T}\right)}+\left\|g_{1}\right\|_{E_{s}^{2+\alpha}\left(\bar{\Gamma}_{T}\right)}+\left\|g_{2}\right\|_{E_{s-1}^{1+\alpha}\left(\bar{\Gamma}_{T}\right)}\right] .
$$

To estimate the functions $v_{\bar{f}_{1}}^{i}, i=1,2$, with respect to $t$, we consider system (4.30)-(4.34) for the difference $\left[v_{\bar{f}_{1}}^{i}\left(x, t_{1}\right)-v_{\bar{f}_{1}}^{i}\left(x, t_{2}\right)\right]$ with the corresponding righthand side and obtain

$$
\begin{gathered}
\sum_{i=1}^{2}\left\|v_{\bar{f}_{1}}^{i}\right\|_{E_{s}^{2+\alpha, \alpha, \alpha}\left(\bar{\Omega}_{i T}\right)} \leq \operatorname{const}\left[\sum_{i=1}^{2}\left\|g_{0 i}\right\|_{E_{s-2}^{\alpha, \alpha, \alpha}\left(\bar{\Omega}_{i T}\right)}\right. \\
\left.+\left\|g_{1}\right\|_{E_{s}^{2+\alpha, \alpha, \alpha}\left(\bar{\Gamma}_{T}\right)}+\left\|g_{2}\right\|_{E_{s-1}^{1+\alpha, \alpha, \alpha}\left(\bar{\Gamma}_{T}\right)}\right] .
\end{gathered}
$$

Now we study the action of the operator $\mathcal{L}$ onto $\mathcal{R} \bar{f}_{1}$ :

$$
\begin{aligned}
& \mathcal{L} \mathcal{R} \bar{f}_{1}=\frac{\partial \mathcal{R} \bar{f}_{1}}{\partial t}+k_{1} a(x) \frac{\partial \bar{\theta}_{1}}{\partial n}+k_{1} A_{1}(x)\left[\frac{\partial \bar{\theta}_{1}}{\partial \omega}-\frac{\partial \bar{\theta}_{2}}{\partial \omega}\right] \\
& =\sum_{m \in N} \eta^{m} \frac{\partial s^{m}}{\partial t}+k_{1} a(x) \frac{\partial \bar{\theta}_{1}}{\partial n}+k_{1} A_{1}(x)\left[\frac{\partial \bar{\theta}_{1}}{\partial \omega}-\frac{\partial \bar{\theta}_{2}}{\partial \omega}\right] .
\end{aligned}
$$

Next, making use of the third conditions in (4.26) and (4.27), we have

$$
\sum_{m \in N} \eta^{m} \frac{\partial s^{m}}{\partial t}=\sum_{m \in N_{1}} \eta^{m} \bar{f}_{1}^{m}(x, t)-k_{1} \sum_{m \in N_{1}} A_{1}^{m}\left[\frac{\partial \bar{w}_{1}^{m}}{\partial \omega}-\frac{\partial \bar{w}_{2}^{m}}{\partial \omega}\right] \eta^{m}
$$




$$
\begin{gathered}
-k_{1} \sum_{m \in N_{1}} a^{m} \eta^{m} \frac{\partial \bar{w}_{1}^{m}}{\partial n}-\left.k_{1} \sum_{m \in N_{2}} A_{1}^{m}\left[\frac{\partial \hat{w}_{1}^{m}(z, t)}{\partial \hat{\omega}}-\frac{\partial \hat{w}_{2}^{m}(z, t)}{\partial \hat{\omega}}\right]\right|_{z=Z_{m}^{-1}(x)} \eta^{m} \\
+\sum_{m \in N_{2}} \eta^{m} \hat{f}_{1}^{m}\left(Z_{m}^{-1}(x), t\right)-\left.k_{1} \sum_{m \in N_{2}} a^{m} \eta^{m} \frac{\partial \hat{w}_{1}^{m}(z, t)}{\partial \hat{n}}\right|_{z=Z_{m}^{-1}(x)}=\bar{f}_{1} \\
-k_{1} \sum_{m \in N_{1}} A_{1}^{m}\left[\frac{\partial \bar{w}_{1}^{m}}{\partial \omega}-\frac{\partial \bar{w}_{2}^{m}}{\partial \omega}\right] \eta^{m}-k_{1} \sum_{m \in N_{1}} a^{m} \eta^{m} \frac{\partial \bar{w}_{1}^{m}}{\partial n}-k_{1} \sum_{m \in N_{2}} a^{m} \eta^{m} \\
\times\left.\frac{\partial \hat{w}_{1}^{m}(z, t)}{\partial \hat{n}}\right|_{z=Z_{m}^{-1}(x)}-\left.k_{1} \sum_{m \in N_{2}} A_{1}^{m}\left[\frac{\partial \hat{w}_{1}^{m}(z, t)}{\partial \hat{\omega}}-\frac{\partial \hat{w}_{2}^{m}(z, t)}{\partial \hat{\omega}}\right]\right|_{z=Z_{m}^{-1}(x)} \eta^{m} .
\end{gathered}
$$

We substitute the value of $\sum_{m \in N} \eta^{m} \frac{\partial s^{m}}{\partial t}$ into (4.36) and get

$$
\begin{gathered}
\mathcal{L} \mathcal{R} \bar{f}_{1}=\bar{f}_{1}+k_{1} a(x)\left[\frac{\partial \bar{\theta}_{1}}{\partial n}-\frac{\partial v_{\bar{f}_{1}}^{1}}{\partial n}\right]+k_{1} A_{1}(x)\left[\frac{\partial \bar{\theta}_{1}}{\partial \omega}-\frac{\partial v_{\bar{f}_{1}}^{1}}{\partial \omega}\right] \\
+k_{1} a(x) \sum_{m \in N} \bar{w}_{1}^{m} \frac{\partial \eta^{m}}{\partial n}-k_{1} A_{1}(x)\left[\frac{\partial \bar{\theta}_{2}}{\partial \omega}-\frac{\partial v_{\bar{f}_{1}}^{2}}{\partial \omega}\right]+k_{1} A_{1}(x) \sum_{m \in N}\left[\bar{w}_{1}^{m}-\bar{w}_{2}^{m}\right] \frac{\partial \eta^{m}}{\partial \omega} \\
+k_{1} \sum_{m \in N}\left[A_{1}(x)-A_{1}^{m}\right]\left[\frac{\partial \bar{w}_{1}^{m}}{\partial \omega}-\frac{\partial \bar{w}_{2}^{m}}{\partial \omega}\right] \eta^{m}+k_{1} \sum_{m \in N}\left[a(x)-a^{m}\right] \frac{\partial \bar{w}_{1}^{m}}{\partial n} \eta^{m}+k_{1} \sum_{m \in N_{2}} A_{1}^{m} \\
\times \eta^{m}\left[\frac{\partial \bar{w}_{1}^{m}(x, t)}{\partial \omega}-\frac{\partial \bar{w}_{2}^{m}(x, t)}{\partial \omega}-\left.\frac{\partial \hat{w}_{1}^{m}(z, t)}{\partial \hat{\omega}}\right|_{z=Z_{m}^{-1}(x)}+\left.\frac{\partial \hat{w}_{2}^{m}(z, t)}{\partial \hat{\omega}}\right|_{z=Z_{m}^{-1}(x)}\right] \\
+k_{1} \sum_{m \in N_{2}} a^{m} \eta^{m}\left[\frac{\partial \bar{w}_{1}^{m}(x, t)}{\partial n}-\left.\frac{\partial \hat{w}_{1}^{m}(z, t)}{\partial \hat{n}}\right|_{z=Z_{m}^{-1}(x)}\right] .
\end{gathered}
$$

To evaluate the right-hand side of (4.37), we describe the properties of the functions $\left(\bar{\theta}_{i}-v_{\bar{f}_{1}}^{i}\right), i=1,2$.

Let

$$
U_{i}(x, t):=\bar{\theta}_{i}-v_{\bar{f}_{1}}^{i},(x, t) \in \bar{\Omega}_{i T} .
$$

Then the simple calculations give (if one takes into account (4.16)-(4.21) and (4.31)-(4.33); and puts $\sigma=\sum_{m \in N} \eta^{m} s^{m}$ in (4.17) as we consider $\mathcal{L} \mathcal{R} \bar{f}_{1}$ now)

$$
\begin{gathered}
\Delta_{x} U_{i}=-g_{0 i}(x, t) \text { in } \Omega_{i T}, \\
U_{1}-U_{2}=\sum_{m \in N}\left[A(x)-A^{m}\right] s^{m} \eta^{m} \equiv \bar{g}_{1}(x, t) \text { on } \Gamma_{T},
\end{gathered}
$$




$$
\begin{gathered}
\frac{\partial U_{1}}{\partial n}-k \frac{\partial U_{2}}{\partial n}=A_{2}(x)\left[\frac{\partial U_{1}}{\partial \omega}-\frac{\partial U_{2}}{\partial \omega}\right]-A_{2}(x) \sum_{m \in N}\left[\bar{w}_{1}^{m}-\bar{w}_{2}^{m}\right] \frac{\partial \eta^{m}}{\partial \omega} \\
-\sum_{m \in N}\left[\bar{w}_{1}^{m}-k \bar{w}_{2}^{m}\right] \frac{\partial \eta^{m}}{\partial n}+\sum_{m \in N} \eta^{m}\left[A_{2}(x)-A_{2}^{m}\right]\left[\frac{\partial \bar{w}_{1}^{m}}{\partial \omega}-\frac{\partial \bar{w}_{2}^{m}}{\partial \omega}\right] \\
=A_{2}(x) \frac{\partial \bar{g}_{1}}{\partial \omega}-A_{2}(x) \sum_{m \in N}\left[\bar{w}_{1}^{m}-\bar{w}_{2}^{m}\right] \frac{\partial \eta^{m}}{\partial \omega}-\sum_{m \in N}\left[\bar{w}_{1}^{m}-k \bar{w}_{2}^{m}\right] \frac{\partial \eta^{m}}{\partial n} \\
+\sum_{m \in N} \eta^{m}\left[A_{2}(x)-A_{2}^{m}\right]\left[\frac{\partial \bar{w}_{1}^{m}}{\partial \omega}-\frac{\partial \bar{w}_{2}^{m}}{\partial \omega}\right] \equiv \bar{g}_{2}(x, t) \text { on } \Gamma_{T}, \\
U_{i}=0 \quad \text { on } \quad \Gamma_{i T}, \quad U_{i}(x, 0)=0 .
\end{gathered}
$$

The one-valued solvability of transmission problem (4.38)-(4.41) and the estimates of the solution follow from the results of Theorem 1.1 and Remark 3.1 [8],

$$
\begin{gathered}
\sum_{i=1}^{2}\left\|U_{i}\right\|_{E_{s}^{2+\alpha, \alpha, \alpha}\left(\bar{\Omega}_{i T}\right)} \leq \operatorname{const}\left[\sum_{i=1}^{2}\left\|g_{0 i}\right\|_{E_{s-2}^{\alpha, \alpha, \alpha}\left(\bar{\Omega}_{i T}\right)}\right. \\
\left.+\left\|\bar{g}_{1}\right\|_{E_{s}^{2+\alpha, \alpha, \alpha}\left(\bar{\Gamma}_{T}\right)}+\left\|\bar{g}_{2}\right\|_{E_{s-1}^{1+\alpha, \alpha, \alpha}\left(\bar{\Gamma}_{T}\right)}\right] .
\end{gathered}
$$

As for the estimate of the right-hand side of (4.42), we have got

$$
\sum_{i=1}^{2}\left\|g_{0 i}\right\|_{E_{s-2}^{\alpha, \alpha, \alpha}\left(\bar{\Omega}_{i T}\right)}+\left\|\bar{g}_{1}\right\|_{E_{s}^{2+\alpha, \alpha, \alpha}\left(\bar{\Gamma}_{T}\right)}+\left\|\bar{g}_{2}\right\|_{E_{s-1}^{1+\alpha, \alpha, \alpha}\left(\bar{\Gamma}_{T}\right)} \leq C(T, \lambda)\left\|\bar{f}_{1}\right\|_{E_{s-1}^{1+\alpha, \alpha, \alpha}\left(\bar{\Gamma}_{T}\right)}
$$

with $0<C(T, \lambda)<<1$ as $\lambda \rightarrow 0$.

The proof of (4.43) is based on tedious calculations by using the results of Theorems 3.1, 3.3 and the properties (4.8)-(4.10) of the functions $a(x), A(x)$, $A_{i}(x), i=1,2$.

Here we prove (4.43) only in the case of $\left\|g_{0 i}\right\|_{E_{s-2}^{\alpha, \alpha, \alpha}\left(\bar{\Omega}_{i T}\right)}$, the rest terms are estimated in a similar way. Note that we use essentially inequalities (39)-(43) from Section 5 [3] and their weighted variants and the results of Theorems 3.1 and 3.2. In these inequalities the minor semi-norms of a function were estimated with the major ones with small coefficients. For example, if a function $W(x, t)$ vanishes as $t=0$, then

$$
\langle W\rangle_{x, s, \Omega_{T}}^{(\alpha)} \leq T^{\alpha}[W]_{s, \Omega_{T}}^{(\alpha, \alpha)}, t \in[0, T]
$$

It is easy to check that

$$
\left\|g_{0 i}\right\|_{E_{s-2}^{\alpha, \alpha, \alpha}\left(\bar{\Omega}_{i T}\right)} \leq \operatorname{const}\left(\sup _{m \in N}\left[\left\|\nabla \eta^{m} \nabla \bar{w}_{i}^{m}\right\|_{E_{s-2}^{\alpha, \alpha, \alpha}\left(\bar{\Omega}_{i T}^{m}\right)}+\left\|\bar{w}_{i}^{m} \Delta \eta^{m}\right\|_{E_{s-2}^{\alpha, \alpha, \alpha}\left(\bar{\Omega}_{i T}^{m}\right)}\right]\right.
$$




$$
\begin{gathered}
\left.+\sup _{m \in N_{22}}\left\|\eta^{m} \Delta \bar{w}_{i}^{m}\right\|_{E_{s-2}^{\alpha, \alpha, \alpha}\left(\bar{\Omega}_{i T}^{m}\right)}\right) \\
\sup _{\bar{\Omega}_{i T}^{m}} r^{-s+2}\left|\bar{w}_{i}^{m}\right|\left|\Delta \eta^{m}\right| \leq \operatorname{const} \frac{T^{\alpha}}{\lambda^{2}}\left\langle\bar{w}_{i}^{m}\right\rangle_{t, s, \bar{\Omega}_{i T}^{m}}^{(\alpha)} \\
\leq \operatorname{const} \lambda^{-2} T^{\alpha^{*}}\left\|\bar{f}_{1}^{m}\right\|_{E_{s-1}^{1+\alpha, \alpha, \alpha}\left(\bar{\Gamma}_{T}^{m}\right)} \\
\left\langle\bar{w}_{i}^{m} \Delta \eta^{m}\right\rangle_{x, s-2, \bar{\Omega}_{i T}^{m}}^{(\alpha)} \leq \operatorname{const}\left(T^{\alpha} \lambda^{-2-\alpha}\left\langle\bar{w}_{i}^{m}\right\rangle_{t, s, \bar{\Omega}_{i T}^{m}}^{(\alpha)}+T^{\alpha} \lambda^{-1-\alpha}\left\langle D_{x} \bar{w}_{i}^{m}\right\rangle_{t, s-1, \bar{\Omega}_{i T}^{m}}^{(\alpha)}\right) \\
\leq \operatorname{const} T^{\alpha^{*}}\left(\lambda^{-2-\alpha}+\lambda^{-1-\alpha}\right)\left\|\bar{f}_{1}^{m}\right\|_{E_{s-1}^{1+\alpha, \alpha, \alpha}\left(\bar{\Gamma}_{T}^{m}\right)} \\
\left\langle\bar{w}_{i}^{m} \Delta \eta^{m}\right\rangle_{t, s-2, \bar{\Omega}_{i T}^{m}}^{(\alpha)} \leq \operatorname{const} \lambda^{-2} T^{\alpha^{*}-\alpha}\left\|\bar{f}_{1}^{m}\right\|_{E_{s-1}^{1+\alpha, \alpha, \alpha}\left(\bar{\Gamma}_{T}^{m}\right)}
\end{gathered}
$$

and at last

$$
\left[\bar{w}_{i}^{m} \Delta \eta^{m}\right]_{s-2, \bar{\Omega}_{i T}^{m}}^{(\alpha, \alpha)} \leq \operatorname{const}\left(T^{\alpha^{*}-\alpha} \lambda^{-2-\alpha}+T^{\alpha^{*}} \lambda^{-1-\alpha}\right)\left\|\bar{f}_{1}^{m}\right\|_{E_{s-1}^{1+\alpha, \alpha, \alpha}\left(\bar{\Gamma}_{T}^{m}\right)},
$$

where $\alpha<\alpha^{*}<1$ as before. Moreover, the simple calculations lead to

$$
\left\|\bar{f}_{1}^{m}\right\|_{E_{s-1}^{1+\alpha, \alpha, \alpha}\left(\bar{\Gamma}_{T}^{m}\right)} \leq \operatorname{const}\left(1+\lambda^{\alpha-1}+\lambda^{-2 \alpha}+\lambda^{1-3 \alpha}\right)\left\|\bar{f}_{1}\right\|_{E_{s-1}^{1+\alpha, \alpha, \alpha}\left(\bar{\Gamma}_{T}\right)} .
$$

Thus, we can conclude from inequalities (4.46)-(4.50) that

$$
\begin{gathered}
\sup _{m \in N}\left\|\bar{w}_{i}^{m} \Delta \eta^{m}\right\|_{E_{s-2}^{\alpha, \alpha, \alpha}\left(\bar{\Omega}_{i T}^{m}\right)} \leq \mathrm{const} \frac{T^{\alpha^{*}-\alpha}}{\lambda^{3+\alpha}}\left(1+\lambda T^{\alpha}\right)\left(\lambda+\lambda^{\alpha}+\lambda^{1-2 \alpha}+\lambda^{2-3 \alpha}\right) \\
\times\left\|\bar{f}_{1}\right\|_{E_{s-1}^{1+\alpha, \alpha, \alpha}\left(\bar{\Gamma}_{T}\right)} \equiv C_{1}(\lambda, T)\left\|\bar{f}_{1}\right\|_{E_{s-1}^{1+\alpha, \alpha, \alpha}\left(\bar{\Gamma}_{T}\right)}
\end{gathered}
$$

If we chose the value $T$ such that

$$
T^{\alpha^{*}-\alpha} \lambda^{-3-\alpha}=\nu<<1,
$$

then due to $0<\alpha<1 / 2$,

$$
C_{1}(\lambda, T)=\nu\left(1+\nu^{\frac{\alpha}{\alpha^{*}-\alpha}} \lambda^{\frac{\alpha(3+\alpha)}{\alpha^{*}-\alpha}}\right)\left(\lambda+\lambda^{\alpha}+\lambda^{1-2 \alpha}+\lambda^{2-3 \alpha}\right)<<1
$$

if $\lambda$ vanishes. The term $\sup _{m \in N}\left\|\nabla \eta^{m} \nabla \bar{w}_{i}^{m}\right\|_{E_{s-2}^{\alpha, \alpha, \alpha}\left(\bar{\Omega}_{i T}^{m}\right)}$ is estimated in the same way. Following the arguments from Chapter 4 [22], we can deduce that the "worst" term in $\sup _{m \in N_{22}}\left\|\eta^{m} \Delta_{x} \bar{w}_{i}^{m}\right\|_{E_{s-2}^{\alpha, \alpha, \alpha}\left(\bar{\Omega}_{i T}^{m}\right)}$ is

$$
\sup _{m \in N_{22}}\left\|\left[\frac{\partial^{2} \hat{w}_{i}^{m}}{\partial z_{1} \partial z_{2}} \Psi_{z_{1}}^{m}\left(z_{1}\right)\right]_{z=Z_{m}^{-1}(x)} \eta^{m}\right\|_{E_{s-2}^{\alpha, \alpha, \alpha}\left(\bar{\Omega}_{i T}^{m}\right)}, \quad m \in N_{22}
$$


(the other terms are evaluated either with the arguments below or with the simpler reasonings).

Note that, as it follows from the definition of the spaces $E_{s}^{k+\alpha, \alpha, \alpha},\|\cdot\|_{E_{s}^{k+\alpha, \alpha, \alpha}\left(\bar{\Omega}_{i T}^{m}\right)}$ $\sim\|\cdot\|_{C^{k+\alpha, \alpha, \alpha}\left(\bar{\Omega}_{i T}^{m}\right)}$ if $m \in N_{22}$. The simple calculations and inequalities (4.23), (4.44), (4.50) drive to

$$
\begin{gathered}
\sup _{\bar{\Omega}_{i T}^{m}}\left|\left[\frac{\partial^{2} \hat{w}_{i}^{m}}{\partial z_{1} \partial z_{2}} \Psi_{z_{1}}^{m}\left(z_{1}\right)\right]_{z=Z_{m}^{-1}(x)} \eta^{m}\right| \leq \operatorname{const} T^{\alpha} \lambda^{1-2 \alpha}\left\|\bar{f}_{1}\right\|_{E_{s-1}^{1+\alpha, \alpha, \alpha}\left(\bar{\Gamma}_{T}\right)} ; \quad(4.54) \\
\left\langle\left[\frac{\partial^{2} \hat{w}_{i}^{m}}{\partial z_{1} \partial z_{2}} \Psi_{z_{1}}^{m}\left(z_{1}\right)\right]_{z=Z_{m}^{-1}(x)} \eta^{m}\right\rangle_{t, s-2, \bar{\Omega}_{i T}^{m}}^{(\alpha)} \leq \operatorname{const} \lambda^{1-2 \alpha}\left\|\bar{f}_{1}\right\|_{E_{s-1}^{1+\alpha, \alpha, \alpha}\left(\bar{\Gamma}_{T}\right)} ; \quad(4.55) \\
\left\langle\left[\frac{\partial^{2} \hat{w}_{i}^{m}}{\partial z_{1} \partial z_{2}} \Psi_{z_{1}}^{m}\left(z_{1}\right)\right]_{z=Z_{m}^{-1}(x)} \eta^{m}\right\rangle_{x, s-2, \bar{\Omega}_{i T}^{m}}^{(\alpha)} \leq \operatorname{const}\left[1+\lambda^{-\alpha}\right] T^{\alpha}\left\|\bar{f}_{1}\right\|_{E_{s-1}^{1+\alpha, \alpha, \alpha}\left(\bar{\Gamma}_{T}\right)} ; \\
{\left[\left(\frac{\partial^{2} \hat{w}_{i}^{m}}{\partial z_{1} \partial z_{2}} \Psi_{z_{1}}^{m}\left(z_{1}\right)\right)_{z=Z_{m}^{-1}(x)} \eta^{m}\right]_{s-2, \bar{\Omega}_{i T}^{m}}^{(\alpha, \alpha)} \leq \operatorname{const} \lambda^{1-2 \alpha}\left\|\bar{f}_{1}\right\|_{E_{s-1}^{1+\alpha, \alpha, \alpha}\left(\bar{\Gamma}_{T}\right)}} \\
+\lambda^{1-\alpha}\left\langle\left.\frac{\partial^{2} \hat{w}_{i}^{m}}{\partial z_{1} \partial z_{2}}\right|_{z=Z_{m}^{-1}(x)}\right\rangle_{t, s-2, \bar{\Omega}_{i T}^{m}}^{(\alpha)}
\end{gathered}
$$

To evaluate the last term in the right-hand side of (4.57), we apply the next interpolate inequality from [23]:

$$
\sup _{\bar{Q}}\left|D_{x}^{2} \Phi(x)\right| \leq\left(\|\Phi\|_{C^{2+\alpha}(\bar{Q})}\right)^{\epsilon^{*}}\left(\sup _{\bar{Q}}|\Phi(x)|\right)^{1-\epsilon^{*}},
$$

where $\epsilon^{*} \in(0,1)$ and $\partial Q \in C^{2}$.

$$
\begin{aligned}
& \text { Putting } \Phi(x):=\left[\frac{\partial^{2} \hat{w}_{i}^{m}\left(z, t_{1}\right)}{\partial z_{1} \partial z_{2}}-\frac{\partial^{2} \hat{w}_{i}^{m}\left(z, t_{2}\right)}{\partial z_{1} \partial z_{2}}\right]_{z=Z_{m}^{-1}(x)} \text { and } \epsilon^{*}=\frac{2}{2+\alpha} \text {, we get } \\
& \left\langle\left.\frac{\partial^{2} \hat{w}_{i}^{m}}{\partial z_{1} \partial z_{2}}\right|_{z=Z_{m}^{-1}(x)}\right\rangle_{t, s-2, \bar{\Omega}_{i T}^{m}}^{(\alpha)} \leq \operatorname{const}\left(\left\|\bar{w}_{i}^{m}\right\|_{C^{2+\alpha, \alpha, \alpha}\left(\bar{\Omega}_{i T}^{m}\right)}\right)^{\frac{2}{2+\alpha}}\left(\left\langle\bar{w}_{i}^{m}\right\rangle_{t, s, \bar{\Omega}_{i T}^{m}}^{(\alpha)}\right)^{\frac{\alpha}{2+\alpha}}
\end{aligned}
$$

or due to the results of Theorem 3.2,

$$
\left\langle\left.\frac{\partial^{2} \hat{w}_{i}^{m}}{\partial z_{1} \partial z_{2}}\right|_{z=Z_{m}^{-1}(x)}\right\rangle_{t, s-2, \bar{\Omega}_{i T}^{m}}^{(\alpha)} \leq \operatorname{const} T^{\frac{2\left(\alpha^{*}-\alpha\right)}{2+\alpha}} \lambda^{-2 \alpha}\left\|\bar{f}_{1}\right\|_{E_{s-1}^{1+\alpha, \alpha, \alpha}\left(\bar{\Gamma}_{T}\right)} .
$$


Then we return to inequality (4.57) and get with (4.59) and (4.52) that

$$
\begin{gathered}
{\left[\left(\frac{\partial^{2} \hat{w}_{i}^{m}}{\partial z_{1} \partial z_{2}} \Psi_{z_{1}}^{m}\left(z_{1}\right)\right)_{z=Z_{m}^{-1}(x)} \eta^{m}\right]_{s-2, \bar{\Omega}_{i T}^{m}}^{(\alpha, \alpha)} \leq \operatorname{const}\left[\lambda^{1-2 \alpha}+\nu^{\frac{2}{2+\alpha}} \lambda^{2+\frac{2}{2+\alpha}}\right]} \\
\times\left\|\bar{f}_{1}\right\|_{E_{s-1}^{1+\alpha, \alpha, \alpha}\left(\bar{\Gamma}_{T}\right)^{.}}
\end{gathered}
$$

Thus, (4.45), (4.51) and (4.53), (4.54)-(4.56), (4.60) prove inequality (4.43) for $\left\|g_{0 i}\right\|_{E_{s-2}^{\alpha, \alpha, \alpha}\left(\bar{\Omega}_{i T}\right)}, i=1,2$.

After that, we return to the right-hand side of (4.37) and, as a consequence of (4.42) and (4.43), we have

$$
\begin{aligned}
& \| k_{1} a(x)\left[\frac{\partial \bar{\theta}_{1}}{\partial n}-\frac{\partial v_{\bar{f}_{1}}^{1}}{\partial n}\right]+ k_{1} A_{1}(x)\left[\frac{\partial \bar{\theta}_{1}}{\partial \omega}-\frac{\partial v_{\bar{f}_{1}}^{1}}{\partial \omega}\right]-k_{1} A_{1}(x)\left[\frac{\partial \bar{\theta}_{2}}{\partial \omega}-\frac{\partial v_{\bar{f}_{1}}^{2}}{\partial \omega}\right] \|_{E_{s-1}^{1+\alpha, \alpha, \alpha}\left(\bar{\Gamma}_{i T}\right)} \\
& \leq \operatorname{const} C(\lambda, T)\left\|\bar{f}_{1}\right\|_{E_{s-1}^{1+\alpha, \alpha, \alpha}\left(\bar{\Gamma}_{T}\right)^{.}}
\end{aligned}
$$

The same arguments together with inequalities from Theorems 3.1 and 3.3 allow us to obtain the estimates like (4.61) for the terms $k_{1} \sum_{m \in N} \bar{w}_{1}^{m} \frac{\partial \eta^{m}}{\partial n}$ and $k_{1} A_{1}(x) \sum_{m \in N}\left[\bar{w}_{1}^{m}-\bar{w}_{2}^{m}\right] \frac{\partial \eta^{m}}{\partial \omega}$ in (4.37).

At last, properties (4.8)-(4.10) of the functions $a(x)$ and $A_{1}(x)$ give

$$
\left\|a(x)-a^{m}\right\|_{C^{1+\alpha}\left(\bar{\Gamma}^{m}\right)}+\left\|A_{1}(x)-A_{1}^{m}\right\|_{C^{1+\alpha}\left(\bar{\Gamma}^{m}\right)} \leq \text { const } \lambda .
$$

To evaluate the rest of the terms in (4.37), we use the same reasonings as above and inequalities (4.23), (4.62). Hence we may conclude that

$$
\left\|\mathcal{L} \mathcal{R} \bar{f}_{1}\right\|_{E_{s-1}^{1+\alpha, \alpha, \alpha}\left(\bar{\Gamma}_{T}\right)} \leq\left\|\bar{f}_{1}\right\|_{E_{s-1}^{1+\alpha, \alpha, \alpha}\left(\bar{\Gamma}_{T}\right)}+C(\lambda, T)\left\|\bar{f}_{1}\right\|_{E_{s-1}^{1+\alpha, \alpha, \alpha}\left(\bar{\Gamma}_{T}\right)}
$$

with $0<C(\lambda, T)<<1$, which completes the proof of Lemma 4.1.

The results of Lemma 4.1 mean that there exists an element $\sigma$ which satisfies to (4.22) and $r^{1+\gamma} \sigma \in E_{s}^{2+\alpha, \alpha, \alpha}\left(\bar{\Gamma}_{T}\right), \sigma_{t} \in E_{s-1}^{1+\alpha, \alpha, \alpha}\left(\bar{\Gamma}_{T}\right)$. Then the existence of the functions $\bar{\theta}_{i}(x, t), i=1,2$, from (4.16)-(4.21) in the corresponding weighted classes follows from [8] in the case of the transmission problem,

$$
\begin{gathered}
\frac{\partial^{2} \bar{\theta}_{i}}{\partial x_{1}^{2}}+\frac{\partial^{2} \bar{\theta}_{i}}{\partial x_{2}^{2}}=0 \quad \text { in } \Omega_{i T}, \\
\bar{\theta}_{1}(x, t)-\bar{\theta}_{2}(x, t)=A(x) \sigma \quad \text { on } \Gamma_{T}, \\
\frac{\partial \bar{\theta}_{1}}{\partial n}-k \frac{\partial \bar{\theta}_{2}}{\partial n}+A_{2}(x)\left(\frac{\partial \bar{\theta}_{1}}{\partial \omega}-\frac{\partial \bar{\theta}_{2}}{\partial \omega}\right)=0 \quad \text { on } \Gamma_{T},
\end{gathered}
$$

Journal of Mathematical Physics, Analysis, Geometry, 2014, vol. 10, No. 1 


$$
\left.\frac{\partial \bar{\theta}_{i}}{\partial x_{1}}\right|_{x_{1}=0}=0, \bar{\theta}_{i}(x, t)=0 \quad \text { on } \quad \Gamma_{i T}, \quad \bar{\theta}_{i}(x, 0)=0
$$

We have

$$
\left\|\bar{\theta}_{i}\right\|_{E_{s}^{2+\alpha, \alpha, \alpha}\left(\bar{\Omega}_{i T}\right)} \leq \mathrm{const}\left\|r^{1+\gamma} \sigma\right\|_{E_{s}^{2+\alpha, \alpha, \alpha}\left(\bar{\Gamma}_{T}\right)}, i=1,2 .
$$

Thus, Lemma 4.1 and (4.65) lead to the following results.

Lemma 4.2. Let the conditions of Lemma 4.1 hold, then there is a solution $\left(\bar{\theta}_{1}, \bar{\theta}_{2}, \sigma\right)$ to problem (4.16)-(4.21), and $\bar{\theta}_{i} \in E_{s}^{2+\alpha, \alpha, \alpha}\left(\bar{\Omega}_{i T}\right), r^{1+\gamma} \sigma \in$ $E_{s}^{2+\alpha, \alpha, \alpha}\left(\bar{\Gamma}_{T}\right), \sigma_{t} \in E_{s-1}^{1+\alpha, \alpha, \alpha}\left(\bar{\Gamma}_{T}\right)$.

Now we need the coercive estimates for the solution $\left(\bar{\theta}_{1}, \bar{\theta}_{2}, \sigma\right)$ which give the uniqueness of the solution obtained in Lemma 4.2.

Lemma 4.3. Let the conditions of Lemma 4.1 hold, then for every $t \in[0, T]$

$$
\left\|\left(\bar{\theta}_{1}, \bar{\theta}_{2}, \sigma\right)\right\|_{H_{D}} \leq \mathrm{const}\left\|\bar{f}_{1}\right\|_{E_{s-1}^{1+\alpha, \alpha, \alpha} \bar{\Gamma}_{T}}
$$

with the constant independent of $\bar{f}_{1}$.

P r o o f. The standard Schauder technique and the results of Section 3 on the properties of model problems lead to the a priori estimate

$$
\left\|\left(\bar{\theta}_{1}, \bar{\theta}_{2}, \sigma\right)\right\|_{H_{D}} \leq \mathrm{const}\left[\left\|\bar{f}_{1}\right\|_{E_{s-1}^{1+\alpha, \alpha, \alpha}\left(\bar{\Gamma}_{T}\right)}+\left\langle\bar{\theta}_{1}\right\rangle_{t, s-2, \bar{\Omega}_{1 T}}^{(\alpha)}+\left\langle\bar{\theta}_{2}\right\rangle_{t, s-2, \bar{\Omega}_{2 T}}^{(\alpha)}\right] .
$$

As for the estimates of $\left\langle\bar{\theta}_{i}\right\rangle_{t, s-2, \bar{\Omega}_{i T}}^{(\alpha)}, i=1,2$, we apply inequality (4.24) from Lemma 4.1 in [12] which gives

$$
\begin{gathered}
\left\langle\bar{\theta}_{1}\right\rangle_{t, s-2, \bar{\Omega}_{1 T}}^{(\alpha)}+\left\langle\bar{\theta}_{2}\right\rangle_{t, s-2, \bar{\Omega}_{2 T}}^{(\alpha)} \leq \mathrm{const}\left\|\bar{f}_{1}\right\|_{E_{s-1}^{1+\alpha, \alpha, \alpha}\left(\bar{\Gamma}_{T}\right)} \\
+\operatorname{const}\left(\varepsilon+C_{\varepsilon} T^{\alpha}\right)\left\|\left(\bar{\theta}_{1}, \bar{\theta}_{2}, \sigma\right)\right\|_{H_{D} .}
\end{gathered}
$$

Choosing $\varepsilon$ and $T$ enough small, we deduce from (4.67) and (4.68) inequality (4.66) for $t \in[0, T]$, where $T$ does not depend on the right-hand side of linear problem (4.16)-(4.21).

Now the proof of Theorem 4.1 can be deduced from the results of Lemmas $4.1-4.3$. 


\section{The Nonlinear Problem: the Proof of Theorem 2.1}

The proof of Theorem 2.1 is based on Theorem 4.1 and representation (2.39) of the nonlinear problem. We can rewrite problem (2.39) in the form

$$
\Im \mathbf{z}=\mathrm{F}(\mathbf{z}) \equiv f(x, t)+\mathrm{F}_{1}(\mathbf{z}),
$$

where $\mathbf{z}=\left(\theta_{1}, \theta_{2}, \sigma\right)$, and $\Im$ is the linear operator which is given by the left-hand side of (2.39), $\Im: H_{D} \rightarrow H_{R}$; the vector $f(x, t)$ is constructed with initial data; $\mathrm{F}_{1}(\mathbf{z})$ contains the elements described in Remark 2.4.

As the operator $\Im$ satisfies the conditions of Theorem 4.1, nonlinear problem (5.1) can be represented as

$$
\mathbf{z}=\Im^{-1} f+\Im^{-1} F_{1}(\mathbf{z}) \equiv P(\mathbf{z}) .
$$

Lemma 5.1. Let $B_{d}, B_{d} \subset H_{D}$, be a ball with the center located in the origin and the radius of $d$. For $\mathbf{z} \in B_{d}$, the following estimates hold:

$$
\begin{gathered}
\left\|F_{1}(0)\right\|_{H_{R}} \leq \operatorname{const} T^{\alpha^{*}-\alpha} \\
\left\|F_{1}\left(\mathbf{z}_{1}\right)-F_{1}\left(\mathbf{z}_{2}\right)\right\|_{H_{R}} \leq \mathrm{const}\left(d+T^{\alpha^{*}-\alpha}\right)\left\|\mathbf{z}_{1}-\mathbf{z}_{2}\right\|_{H_{D}},
\end{gathered}
$$

where $0<\alpha<\alpha^{*}<1$.

The proof of Lemma 5.1 repeats all the arguments from Section 5 [29] and is based on the results of Theorems 3.1 and 4.1.

Note that inequalities (5.2) and (5.3) mean that for sufficiently small $T$ and $d$ the nonlinear operator $P(\mathbf{z})$ satisfies the conditions of the fixed point theorem for a contraction operator. Hence, the fixed point of the operator is the solution of problem (2.39), and thus Theorem 2.1 is proved.

\section{Appendix: The Proof of Lemma 3.1}

To prove the first statement in the lemma, note that the inverse Laplace transformation of the function $\frac{1}{\nu+A}$, where $\operatorname{Re} A>0$, is the function $e^{-A t}$ (see, for example, (5.2.(1)) in [2]). Thus,

$$
K\left(x_{1}, t\right)=\int_{-\infty}^{+\infty} e^{i \lambda x_{1}} e^{-\left(A_{1}|\lambda|+i A_{2} \lambda\right) t} d \lambda=\frac{2 A_{1} t}{\left(A_{1} t\right)^{2}+\left(x_{1}-A_{2} t\right)^{2}},
$$

and (3.28) follows immediately from this representation of $K\left(x_{1}, t\right)$. 
Moreover, using this representation for $K\left(x_{1}, t\right)$, it is easy to get

$$
\frac{\partial^{l} K\left(x_{1}, t\right)}{\partial x_{1}^{l}} \rightarrow 0 \text { as }\left|x_{1}\right| \rightarrow \infty, t \in[0, T] \text {, and } l \geq 0,
$$

which proves (3.29) in the case of $l>0$. To calculate integrals (3.29) in the case of $l=0$, we change the variable $\frac{y-A_{2} \tau}{A_{1} \tau}=z$ in the inner integral and obtain

$$
\int_{0}^{t} d \tau \int_{-\infty}^{\infty} K(y, \tau) d y=4 \int_{0}^{t} d \tau \int_{0}^{\infty} \frac{d z}{z^{2}+1}=2 \pi t
$$

It is easy to find $\frac{\partial K\left(x_{1}, t\right)}{\partial x_{1}}$ from the representation of $K\left(x_{1}, t\right)$,

$$
\frac{\partial K\left(x_{1}, t\right)}{\partial x_{1}}=-2 K^{2}\left(x_{1}, t\right)\left(\frac{x_{1}-A_{2} t}{A_{1} t}\right) .
$$

Thus, to estimate $I_{1}$ in (3.30), we use (6.2) and change of the variable $\frac{y-A_{2} \tau}{A_{1} \tau}=z$ in the inner integral. Then

$$
I_{1}=\mathrm{const} \int_{0}^{t} \frac{d \tau}{\tau^{1-\alpha}} \int_{-\infty}^{\infty} \frac{\left|z+\frac{A_{2}}{A_{1}}\right|^{\alpha}}{\left(1+z^{2}\right)^{2}} \leq \mathrm{const} t^{\alpha}
$$

which proves (3.30).

As for inequality (3.31), we use again representation (6.2) and the change of the variable $y-A_{2} \tau=z$ in the integral with respect to $y$,

$$
\begin{gathered}
\left|I_{2}\right| \leq \text { const }\left|\int_{0}^{t} d \tau \int_{0}^{2|\Delta x|} \frac{\left|z+A_{2} \tau\right|^{\alpha} z A_{1} \tau}{\left(z^{2}+A_{1}^{2} \tau^{2}\right)^{2}} d z\right| \\
\leq \operatorname{const}\left(\int_{0}^{t} d \tau \int_{0}^{2|\Delta x|} \frac{z^{1+\alpha} A_{1} \tau}{\left(z^{2}+A_{1}^{2} \tau^{2}\right)^{2}} d z+\int_{0}^{t} d \tau \int_{0}^{2|\Delta x|} \frac{\left|A_{2}\right|^{\alpha} \tau^{1+\alpha} z A_{1}}{\left(z^{2}+A_{1}^{2} \tau^{2}\right)^{2}} d z\right) \\
\equiv \operatorname{const}\left(i_{1}+i_{2}\right) .
\end{gathered}
$$

First, we evaluate $i_{1}$ and change the variable $z^{2}+A_{1}^{2} \tau^{2}=u$ in the integral with respect to $\tau$. Thus, one has

$$
i_{1} \leq \mathrm{const} \int_{0}^{2|\Delta x|} z^{1+\alpha} d z \int_{z^{2}}^{z^{2}+\left(A_{1} t\right)^{2}} \frac{d u}{u^{2}} \leq \mathrm{const} \int_{0}^{2|\Delta x|} z^{\alpha-1} d z=\mathrm{const}|\Delta x|^{\alpha} .
$$


As for the term $i_{2}$, after the change of the variables $z=A_{1} \tau u$ in the integral with respect to $z$ and then $2|\Delta x|\left(A_{1} \tau\right)^{-1}=v$ in the integral with respect to $\tau$, one gets

$$
\begin{gathered}
i_{2} \leq \mathrm{const} \int_{0}^{t} d \tau \tau^{\alpha-1} \int_{0}^{2 \frac{|\Delta x|}{A_{1} \tau}} \frac{u d u}{\left(u^{2}+1\right)^{2}}=\mathrm{const} \int_{0}^{t} \tau^{\alpha-1}\left[1-\frac{1}{1+4|\Delta x|^{2}\left(A_{1} \tau\right)^{-2}}\right] d \tau \\
\leq \text { const }|\Delta x|^{\alpha} \int_{0}^{+\infty} \frac{v^{1-\alpha} d \alpha}{1+v^{2}} \leq \mathrm{const}|\Delta x|^{\alpha}
\end{gathered}
$$

Hence, inequalities (6.3)-(6.5) lead to estimate (3.31).

Finally, to prove (3.32), we calculate the second derivative of the function $K\left(x_{1}, t\right)$ with respect to $x_{1}$

$$
\frac{\partial^{2} K\left(x_{1}, t\right)}{\partial x_{1}^{2}}=-K^{3}\left(x_{1}, t\right)\left[1-3\left(\frac{x_{1}-A_{2} t}{A_{1} t}\right)^{2}\right],
$$

and change the variables $y=z+A_{2} \tau$ in the integral with respect to $y$ and then $\tau=\frac{z}{A_{1} v}$ in the integral with respect to $\tau$. Thus,

$$
\begin{gathered}
\frac{I_{3}}{|\Delta x|} \leq \text { const } \int_{2|\Delta x|}^{+\infty} d z \int_{0}^{t} \frac{\left[z+A_{2} \tau+\Delta x\right]^{\alpha}}{\left(A_{1} \tau\right)^{3}\left(1+z^{2}\left(A_{1} \tau\right)^{-2}\right)}\left|1-3 z^{2}\left(A_{1} \tau\right)^{-2}\right| d \tau \\
=\operatorname{const} \int_{|\Delta x|}^{+\infty} \frac{d z}{z^{2-\alpha}} \int_{0}^{+\infty} \frac{d v}{\left(1+v^{2}\right)^{2}} \leq \mathrm{const}|\Delta x|^{\alpha-1} .
\end{gathered}
$$

These inequalities lead to estimate (3.32) which completes the proof of Lemma 3.1.

\section{References}

[1] D. Ambrose, Well-Posedness of Two-Phase Hele-Shaw Flow without Surface Tension. - European J. Appl. Math. 15 (2004), 597-607.

[2] H. Bateman and A. Erdélyi, Tables of Integral Transforms. Vol. 1. Book Company, INC, New York, Toronto, London, 1954.

[3] B.V. Bazaliy, Stefan Problem for the Laplace Equation with Regard for the Curvature of the Free Boundary. - Ukr. Math. J. 40 (1997), 1465-1484.

[4] B.V. Bazaliy, Classical Solvability of the Free Boundary Hele-Shaw Problem. Ukr. Math. J. 50 (1998), 1452-1462. 
[5] B. V. Bazaliy and A. Friedman, The Hele-Shaw Problem with Surface Tension in a Half-Plane. - J. Diff. Eqs. 216 (2005), 439-469.

[6] E. Di Benedetto and A. Friedman, The Ill-Posed Hele-Shaw and Stefan Problems for Supercoold Water. - Trans. Amer. Math. Soc. 282 (1984), 183-203.

[7] B.V. Bazaliy and N. Vasylyeva, The Muskat Problem with Surface Tension and a Nonregular Initial Interface. - Nonlinear Analysis: Theory, Methods and Applications 74 (2011), 6074-6096.

[8] B.V. Bazaliy and N. Vasylyeva, The Transmission Problem in Domains with a Corner Point for the Laplace Operator in Weighted Hölder Spaces. - J. Diff. Eqs. 249 (2010), 2476-2499.

[9] B. V. Bazaliy and N. Vasylyeva, On the Solvability of a Transmission Problem for the Laplace Operator with a Dynamic Boundary Condition on a Nonregular Interface. - J. Math. Anal. Appl. 393 (2012), 651-670.

[10] J.A. Cima, A.L. Matheson, and W.T. Ross, The Cauchy Transform. Mathematical Surveys and Monographs 125, AMS, 2006.

[11] I.I. Daniliuk, Nonregular Boundary Problems on a Plane. Nauka, Moscow, 2006. (Russian)

[12] S.P. Degtyarev, The Existence of a Smooth Interface in the Nonstationary Elliptic Muskat-Verigin Problem with a Nonlinear Source. — Ukr. Math. Bull. 7 (2010), 301-330.

[13] C. Elliott and J.R. Ockendon, Weak and Variational Methods for Moving Boundary Problem. Pitman, London, 1982.

[14] J. Esher and B.V. Matioc, On the Parabolicity of the Muskat Problem: WellPosedness, Fingering and Stability Results. - Z. Anal. Anwend. 30 (2011), No. 2, 193-218.

[15] J. Esher and G. Simonett, Classical Solutions of Multidimensional Hele-Shaw Models. — SIAM J. Math. Anal. 28 (1997), 1028-1047.

[16] E.I. Hanzawa, Classical Solution of the Stefan Problem. - Tohoku Math. J. 33 (1981), 297-335.

[17] Y.E. Hohlov and S. Howison, The Classification of Solutions in the Free Boundary Hele-Shaw Problem. — Dokl. Acad. Nauk USSR 325 (1992), 1161-1166.

[18] S. Howison, A Note on the Two-Phase Hele-Shaw Problem. - J. Fluid Mech. 409 (2000), 243-249.

[19] L. Jiang and Y. Chen, Weak Formulation of a Multidimensional Muskat Problem. Free Boundary Problems: Theory and Applications, Vol. II (Irsee,1987), 509-513. Pitman Research Notes in Mathematics Seris, 186. Longman, Harlow, 1990.

[20] J.R. King, A.A. Lacey, and J.L. Vazquez, Persestence of Corners in Free Boundaries in Hele-Shaw Flow. - European J. Appl. Math. 6 (1995), 455-490. 
[21] M.V. Krasnoschok, On an Initial-Boundary Value Problem for a Stationary System of the Theory of Elasticity with Additional Dynamic Condition on a Boundary of a Domain. - Transactions of IAMM 21 (2010), 137-150.

[22] O.A. Ladyzhenskaya, V.A. Solonnikov, and N.N. Ural'tseva, Linear and Quasilinear Parabolic Equations. Transl. Math. Monogr. 23 AMS, Providence, RI, 1968.

[23] A. Lundardi, Analytic Semigroups and Optimal Regularity in Parabolic Problems. Progress in NoDEA. 16 Birkhaüser, Verlag, Basel, 1995.

[24] P.B. Mucha, On the Stefan Problem with Surface Tension in the $L_{p}$ Framework. Adv. Diff. Eqs. 10 (2005), No. 8, 861-900.

[25] M. Muskat, Two Fluid Systems in Porous Media. The Encroachment of Water into an Oil Sand. - Physics 5 (1934), 250-264.

[26] F. Otto, Viscous Fingering: an Optimal Bound on the Growth Rate of the Mixing Zone. - SIAM J. Appl. Math. 57 (1997), No. 4, 982-990.

[27] Ja.A. Roitberg and Z.G. Sheftel', General Boundary Value Problems for Elliptic Equations with Discontinuous Coefficients. - Soviet. Math. Dokl. 4 (1963), 231234. (Russian)

[28] M. Siegel, R. Caflisch, and S. Howison, Global Existence, Singular Solutions, and Ill-Posedness for the Muskat Problem. - Comm. Pure and Appl. Math. 57 (2004), 1374-1411.

[29] N. Vasylyeva, On the Solvability of the Hele-Shaw Problem in the Case of Nonsmooth Initial Data in Weighted Hölder Classes. - Ukr. Math. Bull. 2 (2005), No. 3, 323-349.

[30] F. Yi, Local Classical Solution of Muskat Free Boundary Problem. - J. Partial Diff. Eqs. 9 (1996), 84-96.

[31] F. Yi, Global Classical Solution of Muskat Free Boundary Problem. - J. Math. Anal. Appl. 288 (2003), 442-461. 